Rule Systems for Run-time Monitoring: from EAGLE to RULER

Barringer, Howard and Rydeheard, David and Havelund, Klaus

2010

MIMS EPrint: 2010.55

Manchester Institute for Mathematical Sciences

School of Mathematics

The University of Manchester

\footnotetext{
Reports available from: http://eprints.maths.manchester.ac.uk/

And by contacting: The MIMS Secretary

School of Mathematics

The University of Manchester

Manchester, M13 9PL, UK
} 


\title{
Rule Systems for Run-time Monitoring: from EAGLE to RULER
}

\author{
HOWARD BARRINGER and DAVID RYDEHEARD, School of Computer \\ Science, University of Manchester, Oxford Road, Manchester, M13 9PL, UK. \\ E-mail: howard.barringer@manchester.ac.uk; david.rydeheard@manchester.ac.uk
}

\author{
KLAUS HAVELUND*, NASA's Jet Propulsion Laboratory, California Institute \\ of Technology, Pasadena, CA 91109, USA. \\ E-mail: klaus.havelund@jpl.nasa.gov
}

\begin{abstract}
In Barringer et al. (2004, Vol. 2937, LNCS), EAGLE was introduced as a general purpose rule-based temporal logic for specifying run-time monitors. A novel interpretative trace-checking scheme via stepwise transformation of an EAGLE monitoring formula was defined and implemented. However, even though EAGLE presents an elegant formalism for the expression of complex trace properties, EAGLE's interpretation scheme is complex and appears difficult to implement efficiently. In this article, we introduce RULER, a primitive conditional rule-based system, which has a simple and easily implemented algorithm for effective run-time checking, and into which one can compile a wide range of temporal logics and other specification formalisms used for run-time verification. As a formal demonstration, we provide a translation scheme for linear-time propositional temporal logic with a proof of translation correctness. We then introduce a parameterized version of RULER, in which rule names may have rule-expression or data parameters, which then coincides with the same expressivity as EAGLE with data arguments. RULER with just rule-expression parameters extend the expressiveness of RULER strictly beyond the class of context-free languages. For the language classes expressible in propositional RULER, the addition of rule-expression and data parameters enables more compact translations. Finally, we outline a few simple syntactic extensions of 'core' RULER that can lead to further conciseness of specification but still enabling easy and efficient implementation.
\end{abstract}

Keywords: Run-time verification, rule systems, temporal logic, grammars.

\section{Introduction}

In earlier work, the rule-based temporal logic EAGLE [5] was developed as a generalization of the plethora of logics which have been used for the specification of behavioural system properties and which can be dynamically checked either on-line throughout an execution of the system or off-line over an execution trace of the system. We showed that EAGLE supported future and past time logics, interval logics, extended regular expressions, state machines, logics for real-time and data constraints and temporal-based logics for stochastic behaviour.

The EAGLE logic is a restricted first-order, fixed-point, linear-time temporal logic with chop (concatenation) over finite traces. As such, the logic is highly expressive and, not surprisingly, EAGLE's satisfiability (validity) problem is undecidable; checking satisfiability in a given model, however, is decidable and that is what is required for run-time verification. The syntax and semantics of EAGLE are succinct. There are four primitive temporal operators: $\bigcirc$-next, $\odot$ - previously, - - concatenation and ;-chop (overlapping concatenation, or sequential composition). Temporal equations can be

*The work of this author was carried out at the Jet Propulsion Laboratory, California Institute of Technology, under a contract with the National Aeronautics and Space Administration.

Vol. 20 No. 3, () The Author, 2008. Published by Oxford University Press. All rights reserved.

For Permissions, please email: journals.permissions@oxfordjournals.org

Published online 21 November 2008 doi:10.1093/logcom/exn076 
used to define schema for temporal formulas, where the temporal predicates may be parameterized by data as well as by EAGLE formulas. The usual Boolean logical connectives exist. For example, the linear-time $\square, \diamond, \mathcal{U}$ and $\mathcal{S}$ (always, sometime, until and since) temporal operators can be introduced through the following equational definitions.

$$
\begin{aligned}
& \max A l \text { ways }(\text { Form } F)=F \wedge \bigcirc \operatorname{Always}(F) \\
& \min \operatorname{Sometime}(\operatorname{Form} F)=F \vee \bigcirc \operatorname{Sometime}(F) \\
& \min \operatorname{Until}\left(\operatorname{Form} F_{1}, \text { Form } F_{2}\right)=F_{2} \vee\left(F_{1} \wedge \bigcirc \operatorname{Until}\left(F_{1}, F_{2}\right)\right) \\
& \min \operatorname{Since}\left(\operatorname{Form} F_{1}, \text { Form } F_{2}\right)=F_{2} \vee\left(F_{1} \wedge \bigcirc \operatorname{Since}\left(F_{1}, F_{2}\right)\right)
\end{aligned}
$$

The qualifiers max and min indicate the positive and, respectively, negative interpretation that is to be given to the associated temporal predicate at trace boundaries-corresponding to maximal and minimal solutions to the equations. Thus $\bigcirc$ Always $(p)$ is defined to be true in the last state of a given trace, whereas $\bigcirc \operatorname{Until}(p, q)$ is false in the last state. The formula Always $(p)$ will therefore hold on a finite sequence from, say index $i$, if and only if $p$ holds in every state from index $i$ up to and including the final state. If the formula Until $(p, q)$ holds at index $i$ then $q$ must be true at some state with index $j \geq i$ and $p$ true on all states from $i$ up to but not including $j .{ }^{1}$

Even without data parametrization, the primitive concatenation temporal operators together with the recursively defined temporal predicates take the logic into the world of context-free expressivity thus enabling simple grammatical-like specification of parenthesis, call-return or login-logout matching. Assume call and return are propositions denoting procedure call and return events. The temporal formula Match(call, return) where

$$
\begin{aligned}
& \min \operatorname{Match}(\text { Form } C, \text { Form } R)= \\
& (C \cdot \operatorname{Match}(C, R) \cdot R \cdot \operatorname{Match}(C, R)) \vee \operatorname{Empty}()
\end{aligned}
$$

with Empty() true just on the empty sequence captures the behaviour that every call has a matching return - a call may be followed by a (possibly empty) sequence of matched calls and returns, followed a return, followed by another (possibly empty) sequence of calls and returns. Parametrization of temporal predicates by data values allows us to define real-time and stochastic logical operators. To address real-time, for example, we assume that EAGLE is monitoring time-stamped states, where the state contains a variable clock holding the associated real time. Then it becomes straightforward to define real-time qualified temporal operators such as happens before real time $u$.

$$
\begin{aligned}
& \text { min HappensBefore }(\text { Form } F \text {, double } u)= \\
& \text { clock }<u \wedge(F \vee(\neg F \wedge \bigcirc \text { HappensBefore }(F, u)))
\end{aligned}
$$

It should be clear how more complex real-time, and even probabilistic, temporal operators can be recursively defined.

We continue to believe that EAGLE presents a natural rule/equation-based language for defining, even programming, monitors for complex temporal behavioural patterns. EAGLE is, however, expressively rich and in general this comes with a potentially high computational cost, practically speaking. So one might ask whether EAGLE presents the most appropriate set of primitive temporal operators for run-time monitoring. The non-deterministic concatenation operator, as used above in the matching parentheses example, requires considerable care in use. In order to achieve the

\footnotetext{
${ }^{1}$ Arguments for using other interpretations over finite traces have been put forward. However, we have found that this simple interpretation has been adequate for our monitoring purposes.
} 
expected temporal behaviour pattern, the formulas passed to Match should specify single state sequences. If that is not the case, the concatenation operator may choose an arbitrary cut point, and therefore skip unmatched $C \mathrm{~s}$ or $R \mathrm{~s}$ in order to give a positive result. Later work [2] developed such arguments further and proposed a variety of deterministic versions of temporal concatenation and chop for run-time monitoring, using different forms of cut, e.g. left and right minimal, left and right maximal, etc.

With respect to the computational effectiveness of algorithms for EAGLE trace-checking, in Ref. [5] we showed how trace-checking of full EAGLE can be undertaken on a state-by-state basis without recording the full history, even though the logic has the same temporal expressiveness over the past as over the future; basically, our published trace-check algorithm maintains sufficient knowledge about the past in the evolving monitor formulas. Furthermore, we have shown that for restricted subsets, we can achieve close to optimal complexity bounds for monitoring; one such fragment for which we computed complexity results was the LTL (past and future) fragment of EAGLE [6]. However, whilst the trace-checking algorithm for EAGLE, as presented in Ref. [5] and elsewhere, is theoretically elegant, it remains a challenge, following three different attempts, to obtain a good practically efficient implementation. This is not to say the task is impossible, more that we have failed to do so yet.

What was clear to us at the time was that there were some practically useful and efficiently executable subsets of EAGLE. Despite the pleasing features of EAGLE, we still believe we should continue to search for a powerful and simpler 'core' logic, one that is easy and efficient to evaluate for monitoring purposes. To that end, we present in the remainder of this article a lower level rulebased system, RULER. In Section 2, we introduce RULER and a simple evaluation algorithm by example. Section 3 then provides a formal semantic treatment and in the following two sections, we give a formal translation of finite state automata over finite words in propositional RULER, in Section 4, and a translation scheme for compiling propositional temporal logic (with past and future operators) into RULER, in Section 5. Although not presented, translations from other trace descriptions into propositional RULER, e.g. regular expressions, can easily be given. In Section 6, we consider an extension to basic RULER allowing rule names to be parameterized by both rule and data expression. We present, using just rule-expression parameters, examples of context-free and context-sensitive languages and outline the formal translation of any context-free grammar into ruleexpression parameterized RULER. More efficient evaluation can be achieved via encoding with data as well as rules and to that extent we show how context-free temporal logics such as the CaRet temporal logic of nested procedure calls and returns can be compiled into RULER. The article concludes in Section 7 with a brief reflection and indication of further work.

\section{RuLER by example}

A RULER monitoring system, or rule system, comprises a set of rule names, a set of observation names, a collection of named rules, a set of possible initial states (the initial frontier) and a set of terminally excluded rule names. A rule is formed from a condition part (antecedent) and a body part (consequent). The rule's condition may be a conjunctive set of literals, whereas the body is a disjunctive set of literals, a literal being a positive or negative occurrence of a rule name or of an observation name. The idea is that rules can be made active or inactive. For each active rule, if the condition part evaluates to true for the current state (formed from the current observations and previous obligations of the rule system), then the body of the rule defines what rules are active and what observations must hold in the next state. As a simple example, consider the rule named $r$ below 
in the context of some observation named $a$.

$$
r: \multimap a, r
$$

The rule $r$ has a vacuous condition and its body is a conjunctive set containing observation $a$ and rule name $r$. If $r$ is activated at some point in monitoring, $r$ 's body asserts that the observation $a$ must hold in next monitoring state and the rule $r$ must be active again, thus effectively asserting that observation $a$ must hold in all subsequent monitoring states. Consequently, if at some future state $a$ fails to hold then there will be a conflict between the obligations placed by the rule system and the actual stream of observations. In this simple case, the rule system will fail at that particular point.

The body part of a rule may be disjunctive, as in the rule $r^{\prime}$ below.

$$
r^{\prime}: \multimap a, r^{\prime} \mid b
$$

Assume that both $a$ and $b$ are observation names. If the rule $r^{\prime}$ is active in some monitoring step, then the next monitoring step must satisfy one of the possible choices, i.e. that observation $a$ occurs and $r^{\prime}$ is made active again, or that observation $b$ must occur. If neither $a$ nor $b$ hold in that subsequent step, then again there is a conflict between the obligations of the rule system and the stream of actual observations.

Figure 1 outlines a basic algorithm for monitoring a trace of observation states against a set of named rules defined by a rule system. Essentially, the algorithm explores in a breadth-first fashion all the traces allowed by the rule system against the given trace of sets of observations. In a single monitoring step, the algorithm computes a new frontier (a set of sets of observation obligations and rule activations) according to the given input observations and the current frontier of states. An initial frontier is defined by the rule system. The step computation is repeated until either the monitoring input is exhausted or a conflict between the constraints of the rule system and the input has been determined. A breadth-first exploration of traces allowed by the rule system is undertaken in order to avoid backtracking when a conflict between input observations and rule system obligations occurs.

We refer to a set of rule name literals and observation literals as a rule activation state and hence a frontier is a set of rule activation states. Line 1 of the algorithm in Figure 1 creates an initial frontier using the set of initial rule activation states defined by the rule system. Lines 3-8 programme a single evaluation step of the rule system. In line 4, the current set of observations (obtained in line 3 ) is unioned with each of the rule activation states of the frontier. All inconsistent sets are deleted from the frontier and then, in line 5, a monitoring exception is raised if the frontier has become empty indicating failure of the rule system. Lines 6-8 then build the next frontier. For each consistent resultant state, say $s$, in the frontier (from the action at line 4), a set of possible successor states is

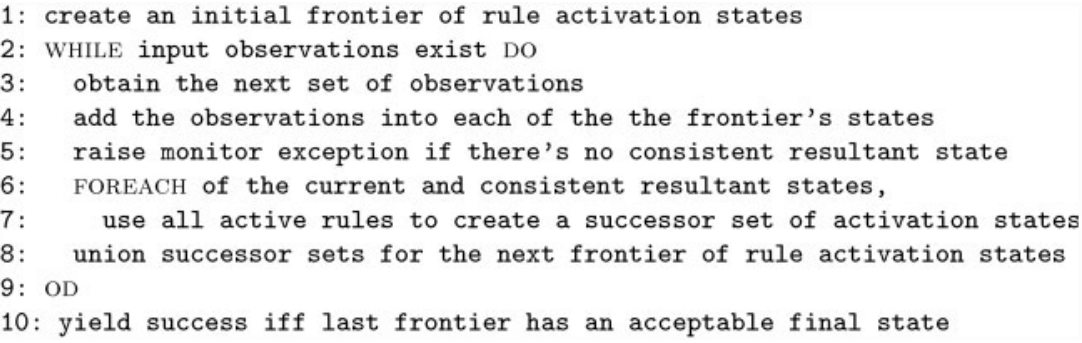

FIGURE 1. The basic monitoring algorithm 
created using the consequents of each applicable rule of $s$ (line 7). The union of the collection of sets of possible successor states, line 8 , then forms the next frontier of rule activation states. The evaluation algorithm re-applies this basic step until either the input is exhausted or a monitoring exception has been raised. For normal termination, line 10, the final frontier is then checked for an acceptable final state, i.e. one not containing a forbidden rule name.

We demonstrate this algorithm in Example 1 where we consider a set of rules that captures both past-time conditions and future-time obligations. We will assume that we wish to monitor some temporal behaviour of a system in terms of two properties, $a$ and $b$. Thus, we arrange for the system to be instrumented to produce a sequence of observation states and that the letters $a$ and $b$ denote particular propositions over an observation state. In effect, we will treat an observation trace as a sequence of consistent sets of literals.

\section{EXAMPLE 1}

We wish to monitor the constraint that whenever property $a$ occurs both now and in the immediate previous state then $b$ must occur as a later observed property. We can characterize this by the linear-time temporal logic formula $\square((a \wedge \odot a) \Rightarrow \oplus b)$ where $\oplus$ is the strict 'eventually in the future' temporal operator, or using the EAGLE temporal predicates defined in Section 1 by the monitoring formula Always $((a \wedge \bigodot a) \Rightarrow \bigcirc$ Sometime $(b))$. In RULER the following set of rules characterizes the required temporal behaviour

$$
\begin{array}{lll}
r_{0}: \multimap r_{0}, r_{1}, r_{3} & r_{1}: a \multimap r_{2} & r_{2}: \\
r_{3}: a, r_{2} \rightarrow b \mid \neg b, r_{4} & r_{4}: \multimap b \mid \neg b, r_{4} &
\end{array}
$$

assuming that the monitoring algorithm starts with an initial set of rule activation sets as $\left\{\left\{r_{0}, r_{1}, r_{3}\right\}\right\}$. Rule $r_{0}$ acts as a generator rule; it ensures persistent activity of itself together with $r_{1}$ and $r_{3}$, i.e. the three rules are always to be active. The empty rule $r_{2}$ is used to represent the temporal constraint $\odot a$ (hence it is initially inactive). The rule $r_{1}$ is then a generator for $r_{2}$ and can be viewed as the temporal rule 'if we have $a$ now then next we have previously $a$ '. Rule $r_{4}$ captures the obligation $₫ b$, either $b$ holds in the next observation state or $\neg b$ holds together with a continued obligation to $₫ b$.

In Table 1 below, we show 8 cycles of the evaluation algorithm on a series of observations for the above rule set. Step 0 corresponds to the initial step of the algorithm (for which we assume no input) in which the frontier of possible rule activation states is initialized to the initial frontier of the rule system (entry in column headed Rule activations); the column headed Resultant states then holds the frontier with the observation set added across it (with inconsistent sets removed). The frontier for step 1 (in column 3 ) is computed as follows. There is just one state in the resultant frontier of step 0 . It has rules $r_{0}, r_{1}$ and $r_{3}$ active. Rule $r_{0}$ unconditionally requires $r_{0}, r_{1}$ and $r_{3}$ to be present in the next step. The condition of rule $r_{1}$ does not hold ( $a$ is not present in the resultant state), therefore there are no obligations from this rule. Similarly, $r_{2}$ does not generate any obligations for the next step. Hence the frontier for step 1 remains as given. For step 1, we have input observations of $a$ and $b$. These observations are consistent with the obligated rule activation frontier for step 1 and hence can be added to each state in the frontier yielding the set in column 4. This resultant state set is used to generate the obligations for the next step. This time since $a$ is in the state, the rule $r_{1}$ will now give rise to rule $r_{2}$ in the rule activations for step 2 alongside $r_{0}, r_{1}$ and $r_{3}$ unconditionally generated by rule $r_{0}$, as before. Moving forward to step 4 where we can see that both $a$ and $\odot a$ are now true-in the resultant state, both $a$ and $r_{2}$ are present—and hence rule $r_{3}$ generates two possibilities for step 5 .

\footnotetext{
${ }^{2}$ The absence of $r_{2}$ from this set gives $\neg r_{2}$ a positive interpretation; this is not the case, however, for observation literals $a$ and $b$ where absence is taken as meaning 'undetermined'.
} 
TABLE 1. Steps of a rule system evaluation

\begin{tabular}{llll}
\hline Step & Obs. & Rule activations & Resultant states \\
\hline 0 & \{\} & $\left\{\left\{r_{0}, r_{1}, r_{3}\right\}\right\}$ & $\left\{\left\{r_{0}, r_{1}, r_{3}\right\}\right\}$ \\
1 & $\{a, b\}$ & $\left\{\left\{r_{0}, r_{1}, r_{3}\right\}\right\}$ & $\left\{\left\{a, b, r_{0}, r_{1}, r_{3}\right\}\right\}$ \\
2 & $\{\neg a, b\}$ & $\left\{\left\{r_{0}, r_{1}, r_{2}, r_{3}\right\}\right\}$ & $\left\{\left\{\neg a, b, r_{0}, r_{1}, r_{2}, r_{3}\right\}\right\}$ \\
3 & $\{a, b\}$ & $\left\{\left\{r_{0}, r_{1}, r_{3}\right\}\right\}$ & $\left\{\left\{a, b, r_{0}, r_{1}, r_{3}\right\}\right\}$ \\
4 & $\{a, b\}$ & $\left\{\left\{r_{0}, r_{1}, r_{2}, r_{3}\right\}\right\}$ & $\left\{\left\{a, b, r_{0}, r_{1}, r_{2}, r_{3}\right\}\right\}$ \\
5 & $\{\neg a, \neg b\}$ & $\left\{\left\{b, r_{0}, r_{1}, r_{2}, r_{3}\right\},\left\{\neg b, r_{0}, r_{1}, r_{2}, r_{3}, r_{4}\right\}\right\}$ & $\left\{\left\{\neg a, \neg b, r_{0}, r_{1}, r_{2}, r_{3}, r_{4}\right\}\right\}$ \\
6 & $\{a, \neg b\}$ & $\left\{\left\{b, r_{0}, r_{1}, r_{3}\right\},\left\{\neg b, r_{0}, r_{1}, r_{3}, r_{4}\right\}\right\}$ & $\left\{\left\{a, \neg b, r_{0}, r_{1}, r_{3}, r_{4}\right\}\right\}$ \\
7 & $\{\neg a, b\}$ & $\left\{\left\{b, r_{0}, r_{1}, r_{2}, r_{3}\right\},\left\{\neg b, r_{0}, r_{1}, r_{2}, r_{3}, r_{4}\right\}\right\}$ & $\left\{\left\{\neg a, b, r_{0}, r_{1}, r_{2}, r_{3}\right\}\right\}$ \\
8 & $\{\neg a, \neg b\}$ & $\left\{\left\{r_{0}, r_{1}, r_{3}\right\}\right\}$ & $\left.\left\{\neg a, \neg b, r_{0}, r_{1}, r_{3}\right\}\right\}$ \\
\hline
\end{tabular}

At step 5, the choice with $b$ holding true conflicts with the observation in step 5 and therefore is eliminated from the resultant states (entry in column 4). Rule $r_{4}$ is thus active and remains activated until step 7 when $b$ is observed to hold thus conflicting with the requirement of $\neg b$ together with $r_{4}$. But how do we determine whether any generated temporal existential obligations, such as $\oplus b$, have indeed been satisfied? The rule system structure records, via the forbidden rule name set, those rule names that correspond to such obligations and then, at the end of monitoring, the monitoring algorithm checks whether the final set of merged observation and rule activation states contains states without those recorded rules active. If there are no such states, then the given (finite) observation trace fails to satisfy the rule set. If there is at least one of the possible final states not containing such recorded rule names, the observation trace satisfies the rule set. The approach is similar to that of the minimal and maximal rule interpretations used in EAGLE. For the above example, rule name $r_{4}$ is specified as a (terminally) forbidden rule name. The final set of merged observation and rule activation states (resultant states, step 8 in the table) has just one possible state that does not contain the forbidden rule $r_{4}$. Hence the given sequence of observations satisfies the given rule set.

The rule set in fact contained an optimization; the choices appearing in rules $r_{3}$ and $r_{4}$ were made deterministic, either $b$ or $\neg b \wedge \ldots$. The determinization thus reduced the number of possible successor states that are generated at any one time. For example, if the rules $r_{3}$ and $r_{4}$ had been defined as

$$
r_{3}: a, r_{2} \rightarrow b\left|r_{4} \quad r_{4}: \multimap b\right| r_{4}
$$

the rule activations for step 7 would be $\left\{\left\{b, r_{0}, r_{1}, r_{2}, r_{3}\right\},\left\{r_{0}, r_{1}, r_{2}, r_{3}, r_{4}\right\}\right\}$, yielding merged states $\left\{\left\{\neg a, b, r_{0}, r_{1}, r_{2}, r_{3}\right\},\left\{\neg a, b, r_{0}, r_{1}, r_{2}, r_{3}, r_{4}\right\}\right\}$. Then, step 8 would have had $\left\{\left\{r_{0}, r_{1}, r_{3}\right\},\left\{b, r_{0}, r_{1}, r_{3}\right\}\right.$, $\left.\left\{r_{0}, r_{1}, r_{3}, r_{4}\right\}\right\}$ for rule activations and $\left\{\left\{\neg a, \neg b, r_{0}, r_{1}, r_{3}\right\},\left\{\neg a, \neg b, r_{0}, r_{1}, r_{3}, r_{4}\right\}\right\}$ for its merged states, one of which does not contain the noted forbidden rule $r_{4}$ and so the observation trace, as is to be expected, satisfies the rule set.

\section{EXAMPLE 2}

A common class of notations for specifying behaviour is variants of state machines. In the following we illustrate how state machines, as presented in RMOR [15], can be translated to RULER. ${ }^{3}$ Suppose that we want to express the following property as a state machine: "every $a$ must be followed by $b$ without any $c$ in between'. We assume that $a, b$ and $c$ are distinct events and that they exclude each other. We assume furthermore that an execution is terminated with a special event $\epsilon$. This property

\footnotetext{
${ }^{3}$ In Section 4, we provide a general translation of finite state automata over finite words into RULER.
} 
can be stated as follows in the RMOR state machine notation and used for specifying and monitoring the behaviour of $\mathrm{C}$ programs.

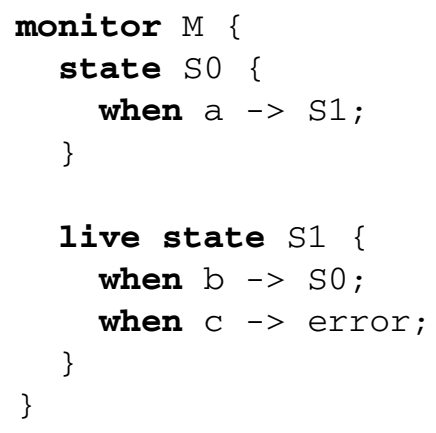

We will here ignore the issue of defining when the events $a, b$ and $c$ are generated. The state machine consists of two states $S 0$, the initial state, and $S 1$. Each state defines a block containing the transitions that leave the state. A live state is a non-final state; monitoring cannot terminate in a live state. In RMOR a state is by default final. A special error state indicates the occurrence of an error, corresponding to entering a non-final state forever. The semantics is that if no transition is enabled in a given moment, we stay in the current state. The following set of rules characterizes the required temporal behaviour in RULER:

$$
\begin{aligned}
& S_{0}: \\
& S_{1}: \\
& r_{1}: S_{0}, a \multimap S_{1} \\
& r_{2}: S_{0}, \neg a \rightarrow S_{0} \\
& r_{3}: S_{1}, b \rightarrow S_{0} \\
& r_{4}: S_{1}, c \rightarrow r_{\text {fail }} \\
& r_{5}: S_{1}, \neg b, \neg c \rightarrow S_{1} \\
& g: \multimap \rightarrow, r_{1}, r_{2}, r_{3}, r_{4}, r_{5} \\
& r_{\text {fail }}: \rightarrow r_{\text {fail }}
\end{aligned}
$$

We here assume that the monitoring algorithm starts with the following initial set of rule activation sets: $\left\{\left\{S_{0}, g, r_{1}, r_{2}, r_{3}, r_{4}, r_{5}\right\}\right\}$, and that the forbidden rule names are $\left\{S_{1}, r_{\text {fail }}\right\}$. That is, a trace fails to match the rule set if either $S_{1}$ (in case $b$ never occurred after an $a$ ) or $r_{\text {fail }}$ (in case a $c$ occurred between an $a$ and a $b$ ) occurs in the final state. The rules $S_{0}$ and $S_{1}$ are memory rules, tracking what state the machine is in at any point in time. Rules $r_{1}$ and $r_{2}$ represent the transitions leaving state $S 0$, whereas rules $r_{3}, r_{4}$ and $r_{5}$ represent the transitions leaving state $S 1$. Rules $r_{2}$ and $r_{5}$ handle the situation where no exiting transition is enabled, and we stay in the current state. This rule set will accept the trace $a b a b \epsilon$, but will reject $a b a \epsilon$ as well as $a b a c b \epsilon$.

\subsection{Inhibiting rule activation}

The informal semantics we have used above has rules being activated in the next step if they appear positively in some applied consequent of some currently applicable rule. In particular, rules that are 
not mentioned in a consequent of some rule cannot be activated by that rule; however, some other rule may indeed activate them. Consider, for example, the contrived (sub)set of rules below.

$$
r_{0}: \multimap r_{2}\left|r_{3} \quad r_{1}: \multimap r_{3}\right| r_{4}
$$

Assume at some stage that $r_{0}$ and $r_{1}$ are activated in the same step. Rule $r_{0}$ therefore generates the partial successor states $\left\{r_{2}\right\}$ and $\left\{r_{3}\right\}$. Rule $r_{1}$ will then extend these states to yield the possible (partial) states $\left\{r_{2}, r_{3}\right\},\left\{r_{2}, r_{4}\right\},\left\{r_{3}\right\}$ and $\left\{r_{3}, r_{4}\right\}$. Suppose it was desired that rules $r_{2}$ and $r_{3}$ were mutually exclusive. One way would be to modify the rules as below.

$$
r_{0}: \multimap r_{2}, \neg r_{3}\left|\neg r_{2}, r_{3} \quad r_{1}: \multimap r_{3}\right| r_{4}
$$

Assuming again both $r_{0}$ and $r_{1}$ active, the possible successor activation sets are now $\left\{r_{2}, \neg r_{3}, r_{4}\right\}$, $\left\{\neg r_{2}, r_{3}\right\}$ and $\left\{\neg r_{2}, r_{3}, r_{4}\right\}$ - since the potential rule activation set $\left\{r_{2}, \neg r_{3}, r_{3}\right\}$ is inconsistent. The negation of a rule should be interpreted as a forced 'non-activation' of the rule.

In the examples above, we indicated how various temporal properties could be translated into collections of low-level single-shot (or step) rules. In a certain sense, rule names can be viewed as propositions denoting temporal subformulas. However, it is important to emphasize that a negated rule name does not correspond to the negation of a subformula that the rule name represents. More strictly, one should view a positive occurrence of a rule name as meaning that the rule will be applied and in doing so will generate possible traces that satisfy the associated subformula. A negative occurrence of a rule name (in the rule activation state) simply means that the rule is NOT applied and hence any constraints imposed by the rule (on the acceptance of traces) will have no effect.

In summary, we can use rules to activate other rules (positive appearance of a rule in a consequent), to not inhibit activation (no mention of a rule in a consequent) and to inhibit activation (negative appearance of a rule in a consequent).

\section{Propositional RuleR trace semantics}

We now present a formalization of propositional rule systems and an evaluation semantics over traces of observations.

Preliminary definitions: Let $X$ denote a set of atoms. We then use $X^{-}$to denote the set of negated atoms of $X$, i.e. $X^{-}=\{\neg x \mid x \in X\}$, and let $X^{ \pm}$denote the set of literals of $X$, i.e. $X \cup X^{-}$. We use the term $X$-literal to refer to a member of $X^{ \pm}$. A set of $X$-literals $L$ is said to be consistent if and only if for any $x \in X$ it is not the case that both $x \in L$ and $\neg x \in L$. Let $L_{X}^{-}$denote the negative closure of $L$ with respect to the atoms $X$, i.e. the set $L \cup\{\neg l \mid l \notin L, l \in X\}$. Given $L S_{1}$ and $L S_{2}$ as sets of consistent sets of literals, the product $L S_{1} \times L S_{2}$ is the set $\left\{l s_{1} \cup l s_{2} \mid l s_{1} \in L S_{1}, l s_{2} \in L S_{2}\right.$, and $l s_{1} \cup l s_{2}$ is consistent $\}$.

\section{DEFINITION 3}

Given disjoint sets of rule names $R$ and observations $O$, a rule $\rho$ is a pair $\langle C, B\rangle$ where $C$, the condition part, is a conjunctive set of $(R \cup O)$-literals, and $B$, the body part, is a disjunctive set of conjunctive sets of $(R \cup O)$-literals. For a given rule $\rho$, we write $C(\rho)$ and $B(\rho)$ for $\rho$ 's condition and body part, respectively. A named rule is then an association $r: \rho$ where $r \in R$ is a rule name and $\rho$ is a rule.

For example, the previous informal presentation of the rule

$$
r_{3}: a, r_{2} \rightarrow b \mid \neg b, r_{4}
$$


is represented as the labelled structure

$$
r_{3}:\left\langle\left\{a, r_{2}\right\},\left\{\{b\},\left\{\neg b, r_{4}\right\}\right\}\right\rangle
$$

where $r_{3}$ is the rule name, the condition body is the set $\left\{a, r_{2}\right\}$ and the body part is the set of sets $\left\{\{b\},\left\{\neg b, r_{4}\right\}\right\}$ representing the two possible choices $\{b\}$ or $\left\{\neg b, r_{4}\right\}$.

\section{DEFINITION 4}

A rule system $R S$ is a tuple $\langle R, O, P, I, F\rangle$ where $R$ and $O$ are, respectively, disjoint sets of rule names and observations, and $P$ is a set of disjointly $R$-named rules over $R$ and $O, I \subseteq 2^{ \pm} \cup R$ is a set of consistent subsets of observation literals and rule names, and $F \subseteq R$ is a set of terminally excluded rule names (rule names that may not appear in the very final monitoring state).

The $I$ component of a rule system defines an initial frontier of possible starting states, i.e. set of required observation literals and rule activations. An initial frontier of $\left\{\left\{a, r_{1}\right\},\left\{\neg b, r_{3}\right\}\right\}$ thus has two possible starting states, one where observation $a$ must be initially true and the rule name $r_{1}$ initially active, and one where observation $b$ must not hold initially but rule $r_{2}$ is initially active.

\section{DEFINITION 5}

A configuration $\gamma$ for a rule system $R S$ is a pair $\langle A, \Theta\rangle$ where $A$ is a consistent set of $R$-literals, called the activity set, and $\Theta$ is a consistent set of $O$-literals, called the observation state. We also write $A(\gamma)$ to denote the activity set of a configuration $\gamma$, similarly $\Theta(\gamma)$ for the observation state.

Of interest now is the interpretation of a set of literals in a configuration. The presence of a positively signed rule name $r$ in the activity set means that the rule $\rho$ associated with $r$ is active. On the other hand, the presence of a negatively signed rule name $r$, or the absence of $r$, in the activity set means that the rule $\rho$ associated with $r$ is not active. For observation atoms, however, undefinedness of an $O$-literal $o$, i.e. the absence of $o$ from the observation state of the configuration, means that the observation literal $o$ may be either true or false.

\section{DEFINITION 6}

Let $R S=\langle R, O, P, I, F\rangle$ be a rule system. A consistent set of literals $L$ from $R S$ holds in a configuration $\gamma$ for $R S$, denoted by $\gamma \models L$, if and only if (i) the set of rule name literals mentioned in $L$ is contained in the negative closure of $A(\gamma)$, i.e. $\left(L-O^{ \pm}\right) \subseteq A(\gamma)_{R}^{-}$, and (ii) observation literals within $L$ are contained in the configuration's set of observations $\left(L-R^{ \pm}\right) \subseteq \Theta(\gamma)$.

Thus, as an example, assume a configuration $\gamma=\left\langle\left\{r_{0}, r_{1}\right\},\{a, \neg b\}\right\rangle$ and consistent sets of literals $L_{1}=\left\{r_{0}, \neg r_{2}, a\right\}$ and $L_{2}=\left\{r_{1}, a, \neg c\right\}$. Assuming that the rule alphabet is $\left\{r_{0}, r_{1}, r_{2}\right\}$ and the observation alphabet is $\{a, b, c\}$, then we have that $\gamma \models L_{1}$ and $\gamma \not \models L_{2}$. $L_{2}$ does not hold in the configuration $\gamma$ because the observation component is quiet on, i.e. does not mention, the observation $c$. We can neither conclude its truth nor its falsity.

We can now define a single step relation over configurations for a given named rule. This relation can then be used to define a single step relation for a rule system.

\section{DEFINITION 7}

An $r: \rho$-step relation $\stackrel{r: \rho}{\longrightarrow}$ between configurations is such that $\gamma \stackrel{r: \rho}{\longrightarrow} \gamma^{\prime}$ if and only if (i) $r \in A(\gamma)$, (ii) $\gamma \models C(\rho)$, and (iii) there is a $\theta \in B(\rho)$ such that $A\left(\gamma^{\prime}\right) \cup \Theta\left(\gamma^{\prime}\right)=\theta$. 
As an example, consider a named rule $r: \rho$ where the rule $\rho$ is the pair $\langle\{a\},\{\{b\},\{c, r\}\}\rangle$ where $a, b$ and $c$ are observation names. Below, we list some possible single steps using this rule.

$$
\begin{aligned}
& \langle\{r\},\{a, b\}\rangle \stackrel{r: \rho}{\longrightarrow}\langle\{r\},\{c\}\rangle \\
& \langle\{r\},\{a, c\}\rangle \stackrel{r: \rho}{\longrightarrow}\langle\{r\},\{c\}\rangle \\
& \langle\{r\},\{a, c\}\rangle \stackrel{r: \rho}{\longrightarrow}\langle\{\},\{b\}\rangle
\end{aligned}
$$

\section{Definition 8}

For any set of rule names $N \subseteq R$, we say $\Gamma^{\prime}$ is an $N$-indexed set of outcome configurations if for each $r \in N$ with $r: \rho \in P, \gamma \stackrel{r: \rho}{\longrightarrow} \Gamma_{r}^{\prime}$.

Consider two named rules $r_{a}:\left\langle\{a\},\left\{\{c\},\left\{a, r_{a}\right\}\right\}\right\rangle$ and $r_{b}:\left\langle\{b\},\left\{\{a\},\left\{b, r_{b}\right\}\right\}\right\rangle$. The following are possible $\left\{r_{a}, r_{b}\right\}$-indexed sets of outcome configurations from the configuration $\left\langle\left\{r_{a}, r_{b}\right\},\{a, b\}\right\rangle$.

$$
\begin{array}{ll} 
& \left\{\left\langle\left\{r_{a}\right\},\{a\}\right\rangle_{r_{a}},\left\langle\left\{r_{b}\right\},\{b\}\right\rangle_{r_{b}}\right\} \\
& \left\{\langle\{\},\{c\}\rangle_{r_{a}},\left\langle\left\{r_{b}\right\},\{b\}\right\rangle_{r_{b}}\right\} \\
& \left\{\left\{\left\{r_{a}\right\},\{a\}\right\rangle_{r_{a}},\langle\{\},\{a\}\rangle_{r_{b}}\right\} \\
& \left\{\langle\{\},\{c\}\rangle_{r_{a}},\langle\{\},\{a\}\rangle_{r_{b}}\right\}
\end{array}
$$

\section{DEFINITION 9}

A single step relation between configurations, $\gamma \longrightarrow \gamma^{\prime}$, holds if and only if $\gamma^{\prime}$ is a consistent union of an $(A(\gamma) \cap R)$-indexed set of outcome configurations from $\gamma$. Note, we assume that an empty union set is treated as being an inconsistent union.

Thus, continuing with the above example, we have

$$
\begin{aligned}
& \left\langle\left\{r_{a}, r_{b}\right\},\{a, b\}\right\rangle \longrightarrow\left\langle\left\{r_{a}, r_{b}\right\},\{a, b\}\right\rangle \\
& \left\langle\left\{r_{a}, r_{b}\right\},\{a, b\}\right\rangle \longrightarrow\left\langle\left\{r_{b}\right\},\{b, c\}\right\rangle \\
& \left\langle\left\{r_{a}, r_{b}\right\},\{a, b\}\right\rangle \longrightarrow\left\langle\left\{r_{a}\right\},\{a\}\right\rangle \\
& \left\langle\left\{r_{a}, r_{b}\right\},\{a, b\}\right\rangle \longrightarrow\langle\{\},\{a, c\}\rangle
\end{aligned}
$$

The single step relation for the rule system can now be used to define the notion of an accepting run of a rule system over a given observation trace. This requires matching observation obligations against actual observations. Note that our semantics requires that if the negation of an observation name is obligated then that negative observation literal must be present in the actual set of observations.

Definition 10

A set of observation literals $X$ is said to match an obligatory set of literals $Y$ if and only if $X \cup Y$ is consistent and $Y \subseteq X$.

Finally, we can define the language accepted by a rule system.

DEFINITION 11 (Language acceptance)

An accepting run of a rule system $R S=\langle R, O, P, I, F\rangle$ on an observation trace $\tau=o_{1} o_{2} \ldots o_{n}$ is a sequence of configurations $\gamma_{1} \gamma_{2} \ldots \gamma_{n}$ such that (i) $\exists s \in I \cdot s=\left(A\left(\gamma_{1}\right) \cup \Theta\left(\gamma_{1}\right)\right)$, (ii) for all $i \in 1 . . n-1$, the set of actual observations, $o_{i}$, matches the set of obligated observations, $\Theta\left(\gamma_{i}\right)$, and $\left\langle A\left(\gamma_{i}\right), \Theta\left(\gamma_{i}\right) \cup\right.$ $\left.o_{i}\right\rangle \longrightarrow \gamma_{i+1}$, and (iii) $A\left(\gamma_{n}\right) \cap F=\{\}$. Hence, the language accepted by a rule system $R S, \mathcal{L}(R S)$, is the set of all finite observation traces $\tau$ accepted by $R S$. Furthermore, we say a rule system $R S$ is violated by an observation trace $\tau$ if $R S$ has no accepting run on $\tau$, alternatively, $\tau \notin \mathcal{L}(R S)$. 
Consider a rule system

$$
\langle\{r\},\{a, b\},\{r:\langle\{\},\{\{a, r\},\{b\}\}\rangle\},\{\{r\}\},\{r\}\rangle .
$$

As there is just one rule, the single step relation $\longrightarrow$ is by definition the relation $\stackrel{r: \rho}{\longrightarrow}$ where $\rho=$ $\langle\{\},\{\{a, r\},\{b\}\}\rangle$, which, in turn, is

$$
\{\langle\langle\{r\}, X\rangle,\langle\{r\},\{a\}\rangle\rangle,\langle\langle\{r\}, X\rangle,\langle\{\},\{b\}\rangle\rangle \mid X \subseteq\{a, b\}\}
$$

The three state observation trace \{\}$\{a\}\{b\}$ has an accepting run, the sequence of configurations

$$
\langle\{r\},\{\}\rangle\langle\{r\},\{a\}\rangle\langle\{\},\{b\}\rangle .
$$

Clearly condition (i) is satisfied. Similarly condition (ii) is satisfied, the input extended configurations are serially related by the single step relation. Finally, the final configuration does not contain a forbidden rule, i.e. \{\} does not contain $r$.

REMARK 12

In Section 2, we outlined the basic steps of a monitoring algorithm for propositional RULER systems. It should be clear that the steps of the informally presented algorithm closely reflect the semantic constructions we have given above. For example, the 'code' at lines 4 and 5 of the algorithm checks for each input state (set of observations) that there is a suitable match with the current obligations of the rule system (embodied in the frontier) - the match condition of part (ii) in the language acceptance definition. Then lines 6-8 of the 'code' compute the successor frontier in accordance with the definition of the single step relation $\longrightarrow$ between configurations. Indeed, if a sufficiently detailed description of the algorithm were presented, it would not be difficult to establish that the algorithm accepts an observation trace $\tau$ for a rule system $R S$ if and only if $\tau \in \mathcal{L}(R S)$.

\section{Finite state automata as rule systems}

In this brief section, we show how non-deterministic finite state automata (NFA), accepting finite words, can be encoded as rule systems.

We define an NFA as a quintuple comprising a finite set of states $S$, a finite alphabet $\Sigma$, a set of labelled transition rules $T \subseteq S \times \Sigma \times S$, an initial state $S_{0}$ and a set of final states $F \subseteq S$. A non-empty finite word $w$ is accepted by NFA if and only if there is a sequence of states $s_{i} \in S$, for $i \in 0 . .|w|$, such that $s_{0}=S_{0}$ and $s_{|w|} \in F$ and for all $i \in 0 . .|w|-1,\left(s_{i}, w_{i}, s_{i+1}\right) \in T$. The empty word is accepted if and only if $S_{0} \in F$.

The components of a rule system $R S(N F A)=\langle R, O, P, I, F\rangle$ that accepts the same language as $N F A$ are defined as below.

$$
\begin{aligned}
& R=\left\{r_{s} \mid s \in S\right\} \\
& O=\Sigma \\
& P=\left\{r_{s}:\left\langle\{\},\left\{\left\{\widehat{\sigma}, r_{t}\right\} \mid(s, \sigma, t) \in T\right\}\right\rangle \mid s \in S\right\} \\
& I=\left\{\left\{\widehat{\sigma}, r_{t}\right\} \mid\left(S_{0}, \sigma, t\right) \in T\right\} \cup\left\{r_{S_{0}} \mid\left(S_{0}, \sigma, t\right) \notin T \vee S_{0} \in F(N F A)\right\} \\
& F=\left\{r_{s} \mid s \in S \wedge s \notin F(N F A)\right\}
\end{aligned}
$$

Each state gives rise to a unique rule name. Similarly, the observation names are defined as the automaton's alphabet. All the transitions from a given state form a single rule whose consequent 
contains the distinguishing disjuncts. We use the notation $\widehat{\sigma_{0}}$ to denoted the (conjunctive) list of RULER observation names $\sigma_{0}, \neg \sigma_{1}, \neg \sigma_{2}, \ldots, \neg \sigma_{|\Sigma|-1}$ for $\sigma_{i} \in \Sigma$ and $\sigma_{i} \neq \sigma_{j}$ when $i \neq j$. The initial frontier is built using the disjuncts of the consequent of the rule corresponding to the initial state of the automaton and using the rule name associated with the initial state of the automaton if the automaton accepts the empty word. The forbidden rules are those rule names corresponding to state names that are not final.

EXAMPLE 13

Consider the finite automaton given by

$$
\begin{aligned}
& S=\{s 0, s 1\} \\
& \Sigma=\{a, b\} \\
& T=\{(s 0, a, s 1),(s 1, c, s 1),(s 1, b, s 0)\} \\
& S_{0}=s 0 \\
& F=\{s 0\}
\end{aligned}
$$

A corresponding rule system is defined by

$$
\begin{aligned}
& R=\left\{r_{s 0}, r_{s 1}\right\} \\
& O=\{a, b\} \\
& P=\left\{r_{s 0}:\left\langle\{\},\left\{\left\{\widehat{a}, r_{s 1}\right\}\right\}\right\rangle, r_{s 1}:\left\langle\{\},\left\{\left\{\widehat{c}, r_{s 1}\right\},\left\{\widehat{b}, r_{s 0}\right\}\right\}\right\rangle\right\} \\
& I=\left\{\left\{r_{s 0}\right\},\left\{\widehat{a}, r_{s 1}\right\}\right\} \\
& F=\left\{r_{s 1}\right\}
\end{aligned}
$$

It is straightforward to establish the following theorem.

THEOREM 14

A word $w$ is accepted by a finite word automaton NFA if and only if the trace of observations formed by $w$ is accepted by the rule system $R S(N F A)$.

\section{Propositional linear temporal logic as a rule system}

We now proceed to show how propositional linear-time temporal logic formulas for monitoring over finite traces can be encoded in RULER. We give an almost direct syntactic transformation, rather than, for example, translating the temporal formula to some form of finite state automaton, which is then encoded in RULER. There are two steps in our translation. The first step is to translate an arbitrary propositional linear-time temporal formula into a collection of universal implications of the form non-strict past formula implies pure future formula, a minor variation of the rule forms used in the executable temporal logic MetateM [4], together with an initial assignment of proposition values. The proof that this is possible is based on the 'Separation Result' of Gabbay, originally 1981 but elaborated in Ref. [12], that shows that any mixed past, present and future linear-time temporal formula can be translated into a semantically equivalent Boolean combination of formulas, each of which depends purely on the past, present or future. We take the translation into the collection of 
universal implications as given. ${ }^{4}$ The second part of the translation, and the part we expand on here, is to show how such separated temporal implications can be represented in RULER. ${ }^{5}$

To provide some informal intuition on the difficulty of a direct translation from mixed past and future temporal formulas, consider the formula $X \mathcal{S}^{-} Y$. In order to determine the truth, or otherwise, of this 'since' formula at some point, say $i$, in a trace, one requires evaluation of the subformulas $X$ and $Y$ at points prior to $i$. If the subformulas $X$ and $Y$ are present or pure past formulas then there is a straightforward translation into RULER that can be used to determine the truth of the 'since' formula. On the other hand, suppose the subformula $X$ is an 'until' formula and hence, as such, it may require evaluation beyond, i.e. in the future of, point $i$. In RULER, this can be handled for a pure future-time formula by using rules that generate obligations on the future part of the trace, but, for the nested case, how are such obligations to be used to provide a truth value for the 'since' formula? Effectively, this would require encoding a complicated mechanism within the direct translation to separate the computation of future obligations from that of past facts. If, however, the original temporal formula has been separated into the 'past implies future' form, we can use the simple translation schemes for the pure past and pure future formulas coupled with a simple scheme for the implicative combination.

\subsection{The pure future part}

The pure future linear-time temporal formulas are built from propositions, the Boolean connectives 'and', 'or', and negation $\left(\wedge, \vee\right.$ and $\neg$, respectively) and strict 'until' and 'unless' operators $\left(\mathcal{U}^{+}\right.$ and $\mathcal{W}^{+}$). All other standard future-time operators are definable from this set. We use the following standard semantics over finite traces of states. Let $\tau=s_{1}, \ldots, s_{i}, \ldots, s_{n}$ where each state $s_{i}$ denotes the subset of propositions that hold true at time $i$. We write $\tau, i \models \phi$ to represent the truth of the temporal formula $\phi$ at time $i$ in the trace $\tau$. An inductive definition of $\models$ is given in Figure 2 .

We assume, without loss of generality, that temporal formulas are in negation normal form $\left(\mathrm{NNF}^{6}\right)$, i.e. negation operators pushed inwards to propositional literals and cancellations applied. Let $\mathrm{WFF}^{+}(\mathrm{Obs})$ denote the set of well-formed strict future-time formulas over proposition alphabet $\mathrm{Obs}$ in NNF and $W F F(\mathrm{Obs})$ denote the set of well-formed future-time formulas over proposition alphabet $O b s$ in NNF (which may include the present, i.e. propositions under no future-time operator). For convenience, we omit the observation alphabet $O b s$ when either it is clear from the context or unimportant.

Figure 3 presents a translation $\vec{T}: W F F \rightarrow$ RuleSystem defined inductively over the structure of the temporal formulas. Let $\phi$ and $\psi$ denote arbitrary members of $W F F$. The base cases of the translation are straightforward. The propositional constant true gives rise to the rule system $\left\langle\left\{r_{. g}\right\},\{\},\left\{r_{. g}\right.\right.$ : $\left.\left.\rightarrow\left\{\left\{r_{. g}\right\}\right\}\right\},\left\{\left\{r_{. g}\right\}\right\},\{\}\right\rangle$ where the initial frontier of states contains just a singleton set with the rule $r_{. g}$ thus placing no constraints on the observation state. The rule $r_{. g}$ is used to keep the rule system active as any length of observation trace should be accepted by the system. On the other hand, false translates to a system with an empty set of initial states, denoting vacuity there are no observation traces that satisfy false. For a proposition $p$, we have $\vec{T}(p)=\left\langle\left\{r_{. g}\right\},\{p\}\right.$,

\footnotetext{
${ }^{4}$ The complexity of the separation translation is exponential in the nesting depth of alternating pairs of until and since temporal operators. However, as we have argued with METATEM [4], the separated temporal implicative rule form is a natural form in which to present temporal specifications.

${ }^{5}$ Fisher's Separated Normal Form (SNF) representation for temporal logic [10] is close to RULER rule forms and an alternative translation to a rule system could be given via SNF. However, we believe our direct translation has interest in its own right and might lead to an easier SNF translation.

${ }^{6}$ Some authors refer to this as positive normal form.
} 


$$
\begin{aligned}
& \tau, i \models \text { true } \\
& \tau, i \not \models \text { false } \\
& \tau, i \models p \quad \text { iff } p \in \tau(i) \\
& \tau, i \models \neg \phi \quad \text { iff } \tau, i \not \models \phi \\
& \tau, i \models \phi \wedge \psi \quad \text { iff } \tau, i \models \phi \text { and } \tau, i \models \psi \\
& \tau, i \models \phi \vee \psi \quad \text { iff } \tau, i \models \phi \text { or } \tau, i \models \psi \\
& \tau, i \models \phi \mathcal{U}^{+} \psi \text { iff there exists } k>i \text { st } \tau, k \models \psi \text { and } \\
& \text { for all } j \text { where } k>j>i \text { we have } \tau, j \models \phi \\
& \tau, i \models \phi \mathcal{W}^{+} \psi \text { iff there exists } k>i \text { st } \tau, k \models \psi \text { and } \\
& \text { for all } j \text { where } k>j>i \text { we have } \tau, j \models \phi \\
& \text { or for all } j \text { where }|\tau| \geq j>i \text { we have } \tau, j \models \phi
\end{aligned}
$$

FIGURE 2. Future linear-time temporal logic semantics

$$
\begin{aligned}
& \vec{T} \text { (true) }=\left\langle\{r . g\},\{\},\left\{r . g: \multimap\left\{\left\{r_{. g}\right\}\right\}\right\},\left\{\left\{r_{. g}\right\}\right\},\{\}\right\rangle \\
& \vec{T}(\text { false })=\langle\{\},\{\},\{\},\{\},\{\}\rangle \\
& \vec{T}(p) \quad=\left\langle\{r . g\},\{p\},\left\{r_{. g}: \multimap\left\{\left\{r_{. g}\right\}\right\}\right\},\left\{\left\{p, r_{. g}\right\}\right\},\{\}\right\rangle \\
& \vec{T}(\neg q) \quad=\left\langle\left\{r_{. g}\right\},\{q\},\left\{r_{. g}: \multimap\left\{\left\{r_{. g}\right\}\right\}\right\},\left\{\left\{\neg q, r_{. g}\right\}\right\},\{\}\right\rangle \\
& \vec{T}(\phi \wedge \psi)=\operatorname{LET}\left\langle R_{\phi}, O_{\phi}, P_{\phi}, I_{\phi}, F_{\phi}\right\rangle=\vec{T}(\phi) \text { AND }\left\langle R_{\psi}, O_{\psi}, P_{\psi}, I_{\psi}, F_{\psi}\right\rangle=\vec{T}(\psi) \\
& \text { IN }\left\langle R_{\phi} \cup R_{\psi}, O_{\phi} \cup O_{\psi}, P_{\phi} \cup P_{\psi}, I_{\phi} \times I_{\psi}, F_{\phi} \cup F_{\psi}\right\rangle \\
& \vec{T}(\phi \vee \psi)=\operatorname{LET}\left\langle R_{\phi}, O_{\phi}, P_{\phi}, I_{\phi}, F_{\phi}\right\rangle=\vec{T}(\phi) \text { AND }\left\langle R_{\psi}, O_{\psi}, P_{\psi}, I_{\psi}, F_{\psi}\right\rangle=\vec{T}(\psi) \\
& \text { IN }\left\langle R_{\phi} \cup R_{\psi}, O_{\phi} \cup O_{\psi}, P_{\phi} \cup P_{\psi}, I_{\phi} \cup I_{\psi}, F_{\phi} \cup F_{\psi}\right\rangle \\
& \vec{T}\left(\phi \mathcal{U}^{+} \psi\right)=\operatorname{LET}\left\langle R_{\phi}, O_{\phi}, P_{\phi}, I_{\phi}, F_{\phi}\right\rangle=\vec{T}(\phi) \text { AND } \\
& \left\langle R_{\psi}, O_{\psi}, P_{\psi}, I_{\psi}, F_{\psi}\right\rangle=\vec{T}(\psi) \\
& \text { IN }\left\langle R_{\phi} \cup R_{\psi} \cup\left\{r_{\phi \mathcal{U}}+\psi\right\}, O_{\phi} \cup O_{\psi}\right. \text {, }
\end{aligned}
$$

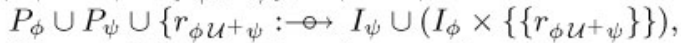

$$
\begin{aligned}
& \left.\left\{\left\{r_{\phi \mathcal{U}^{+} \psi}\right\}\right\}, F_{\phi} \cup F_{\psi} \cup\left\{r_{\phi \mathcal{U}}{ }^{+}\right\}\right\} \\
& \vec{T}\left(\phi \mathcal{W}^{+} \psi\right)=\operatorname{LET}\left\langle R_{\phi}, O_{\phi}, P_{\phi}, I_{\phi}, F_{\phi}\right\rangle=\vec{T}(\phi) \text { AND } \\
& \left\langle R_{\psi}, O_{\psi}, P_{\psi}, I_{\psi}, F_{\psi}\right\rangle=\vec{T}(\psi) \\
& \text { IN }\left\langle R_{\phi} \cup R_{\psi} \cup\left\{r_{\phi \mathcal{W}+\psi}\right\}, O_{\phi} \cup O_{\psi}\right. \text {, }
\end{aligned}
$$

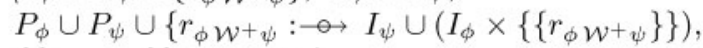

$$
\begin{aligned}
& \left.\left\{\left\{r_{\phi} \mathcal{W}+\psi\right\}\right\}, F_{\phi} \cup F_{\psi}\right\rangle
\end{aligned}
$$

FIGURE 3. Translation of future linear-time temporal formulas

$\left.\left\{r_{. g}: \multimap\left\{\left\{r_{. g}\right\}\right\}\right\},\left\{\left\{p, r_{. g}\right\}\right\},\{\}\right\rangle$, indicating a rule system with an observation atom $p$ with an initial frontier containing the set $\left\{p, r_{. g}\right\}$. The rule $r_{. g}$ is included as any observation trace that has $p$ present initially is acceptable, therefore the rule system must keep active until the end of the observation trace. Negated atoms translate in a similar way, apart from the initial frontier containing the set comprising the negated atom and the rule $r_{. g}$. The logical conjunction (disjunction) of formulas $\phi$ and $\psi$ translate to a product (union) operation that can be defined for rule systems, as defined in Figure 3 . This leaves the most interesting part of the translation, namely an 'until' formula $\phi \mathcal{U}^{+} \psi$. Recall that the semantics of the strict 'until' operator gives the temporal equivalence $\phi \mathcal{U}^{+} \psi \Leftrightarrow \bigcirc\left(\psi \vee\left(\phi \wedge\left(\phi \mathcal{U}^{+} \psi\right)\right)\right)$. This recursive definition is then directly encoded using rules. For ease of understanding, we have subscripted the rule names by the sub-formulas they represent. As the 'until' operator has a strong interpretation, requiring its second argument to be satisfied, the associated rule name for an 'until' 
formula must be included in the $F$ set of the rule system. As might be expected, the translation of an 'unless' formula differs from the 'until' translation just in the non-inclusion of the rule for the 'unless' formula in the $F$ set.

\section{EXAMPLE 15}

Assume $a, b, c$ and $d$ are atomic propositions. The translation of $a \mathcal{U}^{+} b$ yields a rule system with rule names $R=\left\{r_{. g}, r_{a \mathcal{U}^{+}} b\right.$, observation names $O=\{a, b\}$, rules $P$ as

$$
\begin{aligned}
& r_{. g}: \multimap r_{. g} \\
& r_{a \mathcal{U}^{+} b}: \stackrel{\rightarrow}{\rightarrow}, r_{. g} \mid a, r_{. g}, r_{a \mathcal{U}^{+} b}
\end{aligned}
$$

initial frontier $I=\left\{\left\{r_{a \mathcal{U}^{+}} b\right\}\right\}$ and forbidden rules $F=\left\{r_{a \mathcal{U}^{+} b}\right\}$. Similarly, the translation of $a \wedge$ $\left(c \mathcal{W}^{+} d\right)$ yields a rule system with $R=\left\{r_{. g}, r_{c} \mathcal{W}^{+} d\right\}, O=\{a, c, d\}$, rules $P$ as

$$
\begin{aligned}
& r_{. g}: \multimap r_{. g} \\
& r_{c \mathcal{W}^{+} d}: \stackrel{\hookrightarrow}{\rightarrow}, r_{. g} \mid c, r_{. g}, r_{c} \mathcal{W}^{+} d
\end{aligned}
$$

initial frontier $I=\left\{\left\{a, r_{. g}, r_{c \mathcal{W}} \mathcal{W}_{d}\right\}\right\}$ and an empty forbidden rule set $F$. Thus the translation of $\left(a \mathcal{U}^{+} b\right) \mathcal{U}^{+}\left(a \wedge\left(c \mathcal{W}^{+} d\right)\right)$ yields the rule system

$$
\left\langle\left\{r_{. g}, r_{0}, r_{1}, r_{2}\right\},\{a, b, c, d\},\left\{\begin{array}{l}
r_{. g}: \multimap r_{. g} \\
r_{0}: \multimap b, r_{. g} \mid a, r_{. g}, r_{0} \\
r_{1}: \multimap d, r_{. g} \mid c, r_{. g}, r_{1} \\
r_{2}: \multimap a, r_{. g}, r_{1} \mid r_{0}, r_{2}
\end{array}\right\},\left\{\left\{r_{2}\right\}\right\},\left\{r_{0}, r_{2}\right\}\right\rangle
$$

where

$$
r_{0}=r_{a \mathcal{U}^{+} b}, r_{1}=r_{c \mathcal{W}^{+} d}, r_{2}=r_{\left(a \mathcal{U}^{+} b\right) \mathcal{U}^{+}\left(a \wedge\left(c \mathcal{W}^{+} d\right)\right)}
$$

The correctness of the translation defined in Figure 3 is given in the following result.

\section{THEOREM 16}

For any NNF formula $\phi \in W F F(O b s)$ and finite observation trace $\tau$ over observation propositions Obs, the suffix of $\tau$ from index $i$, denoted by $\tau^{(i)}$, is in the language $\mathcal{L}(\vec{T}(\phi))$ if and only $\tau, i \models \phi$.

PROOF. We proceed by induction over the structure of the formula $\phi$.

(i) $\phi$ is true. The generated rule system has just one rule of the form

$$
r_{. g}: \multimap r_{. g}
$$

with an initial frontier $I$ of $\left\{\left\{r_{. g}\right\}\right\}$ and an empty forbidden rule set $F$. This rule has an empty condition part, and hence always generates obligations when the rule is active. Furthermore, the rule's obligation set places no constraint on observations, it only ensures that the rule is active again for subsequent application. As there are also no constraints on rule names active in any final configuration, any finite observation trace will be accepted by the rule system. This corresponds to all possible linear observation sequences that are models for the temporal formula true. The desired result follows since true is independent of the past.

(ii) $\phi$ is false. The generated rule system has no rules and an empty initial frontier. There are thus no initial observations that can be matched with the initial frontier, hence the language of the rule system is empty, corresponding to the empty set of models for the temporal formula false. 
(iii) $\phi$ is a proposition $p$. The generated rule system differs from that generated for the formula true in that the initial frontier $I$ also contains the observation proposition $p$, hence requiring it to be present in the initial state of all observation traces accepted by the rule system. The single rule of the system places no further constraints on the observations. Hence the language accepted by the rule system corresponds to the set of traces $\tau$ over $O b s$ such that $\tau, 0 \models p$, and thus, as $p$ is independent of the past, $\tau^{(i)} \in \mathcal{L}(\vec{T}(p))$ if and only if $\tau, i \models p$.

(iv) $\phi$ is $\neg q$ for proposition $q$. The $\operatorname{argument}$ here is similar to (iii) apart from the requirement that $q$ cannot be present initially.

(v) $\phi$ is $X \wedge Y$ for $X, Y \in W F F(O b s)$. Wlog, we assume that the rule name alphabets of $\vec{T}(X)$ and $\vec{T}(Y)$ are disjoint. Consider a trace $\tau$ such that $\tau, i \models X \wedge Y$. Thus by the temporal logic semantics we have that $\tau, i \models X$ and $\tau, i \models Y$. Using the induction hypotheses for the sub-formulas $X$ and $Y$, there are thus accepting runs $\sigma_{X}$ and $\sigma_{Y}$ over $\tau^{(i)}$ of rule systems $\vec{T}(X)$ and $\vec{T}(Y)$, respectively. Let $\sigma_{X Y}$ denote the pointwise union of the two accepting runs (respecting the activation and observation set structure). We argue that $\sigma_{X Y}$ will be an accepting run of the rule system $\vec{T}(X \wedge Y)$ : (a) the initial set $I(\vec{T}(X \wedge Y))$ comprises all the union pairings of the elements of $I(\vec{T}(X))$ and $I(\vec{T}(Y))$, hence, by construction, at least one of the unions will 'hold' initially on $\sigma_{X Y}$; and (b) as the rule name sets of each translation are disjoint, the run can be viewed as the parallel run of rule systems for $X$ and for $Y$.

For the other direction, we consider a trace $\tau$ such that $\tau, i \not \models X \wedge Y$. Assume wlog. that $\tau, i \not \models X$. Thus, by the induction hypothesis, there is no accepting run $\sigma_{X}$ of $\vec{T}(X)$. By the construction of $\vec{T}(X \wedge Y)$ there cannot be an accepting run, for if there were we could project out the run on the rule names of $X$ and thereby construct an accepting run of $\vec{T}(X)$.

(vi) $\phi$ is $X \vee Y$ for $X, Y \in W F F(O b s)$. This is similar to (v).

(vii) $\phi$ is $X \mathcal{U}^{+} Y$ for $X, Y \in W F F(O b s)$. We establish $\tau^{(i)} \in \mathcal{L}\left(\vec{T}\left(X \mathcal{U}^{+} Y\right)\right)$ for any $i \in 1 . .|\tau|$ by a downward induction on $i$ under the overall hypotheses $\tau^{(i)} \in \mathcal{L}(\vec{T}(X))$ and $\tau^{(i)} \in \mathcal{L}(\vec{T}(Y))$ for any $i \in 1 . .|\tau|$.

Base case: This is when $i=|\tau|$. By the temporal logic semantics, $\tau,|\tau| \not \models X \mathcal{U}^{+} Y$ - there is no future. There is, however, no accepting run of $\vec{T}\left(X \mathcal{U}^{+} Y\right)$ of length 1 because $\left\{r_{X \mathcal{U}^{+} Y}\right\}$ must be in $I$ and also a subset of $F$. Hence $\tau^{(|\tau|)} \in \mathcal{L}\left(\vec{T}\left(X \mathcal{U}^{+} Y\right)\right)$ iff $\tau,|\tau| \models X \mathcal{U}^{+} Y$.

Inductive step: We assume $\tau^{(j)} \in \mathcal{L}\left(\vec{T}\left(X \mathcal{U}^{+} Y\right)\right)$ iff $\tau, j \models X \mathcal{U}^{+} Y$ for $\left.j, 1<j \leq \mid \tau\right]$ and show $\tau^{(j-1)} \in \mathcal{L}\left(\vec{T}\left(X \mathcal{U}^{+} Y\right)\right)$ iff $\tau, j-1 \models X \mathcal{U}^{+} Y$. By the overall induction hypothesis for $X$ and $Y$ and the downward induction hypothesis for $X \mathcal{U}^{+} Y$, we claim that $\tau^{(j)} \in \mathcal{L}(\vec{T}(Y \vee(X \wedge$ $\left.\left.\left.X \mathcal{U}^{+} Y\right)\right)\right)$ iff $\tau, j=Y \vee\left(X \wedge X \mathcal{U}^{+} Y\right)$. First observe that there is an accepting run of $\vec{T}(Y \vee$ $\left.\left(X \wedge X \mathcal{U}^{+} Y\right)\right)$ on $\tau^{(j)}$ if there is an accepting run of $\vec{T}(Y)$ on $\tau^{(j)}$ or there is an accepting run on $\tau^{(j)}$ for both $\vec{T}(X)$ and $\vec{T}\left(X \mathcal{U}^{+} Y\right)$; this follows from the fact that the set of sets of initial conditions $I\left(\vec{T}\left(Y \vee\left(X \wedge X \mathcal{U}^{+} Y\right)\right)\right)$ is the same as $I(\vec{T}(Y)) \cup\left(I(\vec{T}(X)) \times I\left(\vec{T}\left(X \mathcal{U}^{+} Y\right)\right)\right)$. By the hypotheses, we thus have $\tau, j \models Y$ or both $\tau, j \models X$ and $\tau, j \models X \mathcal{U}^{+} Y$, and therefore $\tau, j \models Y \vee\left(X \wedge X \mathcal{U}^{+} Y\right)$, which means, by the temporal semantics of $\mathcal{U}^{+}$that $\tau, j-1 \models X \mathcal{U}^{+} Y$. Furthermore, if we have an accepting run of $\vec{T}\left(Y \vee\left(X \wedge X \mathcal{U}^{+} Y\right)\right)$ on $\tau^{(j)}$ then the rule system $\vec{T}\left(X \mathcal{U}^{+} Y\right)$ will have an accepting run on $\tau^{(j-1)}$; the rule $r_{X \mathcal{U}^{+} Y}$ obligates $I(\vec{T}(Y)) \cup$ 
$\left(I(\vec{T}(X)) \times\left\{\left\{r_{X \mathcal{U}^{+} Y}\right\}\right\}\right)$ on the next step, and as $I\left(\vec{T}\left(X \mathcal{U}^{+} Y\right)\right)=\left\{\left\{r_{X \mathcal{U}^{+} Y}\right\}\right\}$, we will have an accepting run on $\tau^{(j-1)}$.

Second, there is no accepting run of $\vec{T}\left(Y \vee\left(X \wedge X \mathcal{U}^{+} Y\right)\right)$ on $\tau^{(j)}$ if there is no accepting run on $\tau^{(j)}$ for $\vec{T}(Y)$ and for at least one of $\vec{T}(X)$ and $\vec{T}\left(X \mathcal{U}^{+} Y\right)$. Hence, by the hypotheses we have $\tau, j \not \models Y \vee\left(X \wedge X \mathcal{U}^{+} Y\right)$ and therefore, by the semantics of $\mathcal{U}^{+}$, we have $\tau, j-1 \not \models$ $X \mathcal{U}^{+} Y$. But if there is no accepting run of $\vec{T}\left(Y \vee\left(X \wedge X \mathcal{U}^{+} Y\right)\right)$ on $\tau^{(j)}$ then there is no accepting run of $\vec{T}\left(X \mathcal{U}^{+} Y\right)$ on $\tau^{(j-1)}$, for if this were not the case we would have an accepting run of $\vec{T}\left(Y \vee\left(X \wedge X \mathcal{U}^{+} Y\right)\right)$ on $\tau^{(j)}$, which is not the case.

Hence the desired result.

(viii) $\phi$ is $X \mathcal{W}^{+} Y$ for $X, Y \in W F F(O b s)$. This case is argued in a similar way to the case for $\mathcal{U}^{+}$apart from the fact that the base case has the formula $X \mathcal{W}^{+} Y$ true on the final observation state with the rule $r_{X \mathcal{W}^{+} Y}$ not forbidden from the final sets of activated rules.

Past-time temporal queries: The pure past-time fragment of linear-time temporal logic is constructed in a mirror fashion to the pure future part, i.e. from propositions, the Boolean connectives $(\wedge, \vee$ and $\neg$ ), and just the temporal operators $\mathcal{S}^{-}$(the strict since, false at the beginning of time) and its weak version $\mathcal{Z}^{-}$(true at the beginning of time). We define the semantics of the $\mathcal{S}^{-}$and $\mathcal{Z}^{-}$temporal operators.

$$
\begin{array}{r}
\tau, i=\phi \mathcal{S}^{-} \psi \text { iff there exists } k \text { st } 1 \leq k<i \text { and } \tau, k \models \psi \text { and } \\
\text { for all } j \text { where } k<j<i \text { we have } \tau, j \models \phi \\
\tau, i=\phi \mathcal{Z}^{-} \psi \text { iff there exists } 1 \leq k<i \text { st } \tau, k \models \psi \text { and } \\
\text { for all } j \text { where } k<j<i \text { we have } \tau, j \models \phi \\
\text { or for all } j \text { where } 1 \leq j<i \text { we have } \tau, j \models \phi
\end{array}
$$

Without loss of generality, we assume that past-time temporal formulas are in negation normal form. Let us first informally consider the translation of pure past-time temporal queries. The temporal equivalence $\phi \mathcal{S}^{-} \psi \Leftrightarrow \odot\left(\psi \vee\left(\phi \wedge\left(\phi \mathcal{S}^{-} \psi\right)\right)\right)$ should serve as a reminder of the semantics that needs to be captured by the translation. The basic idea for handling the past is an old one, namely, we use the translation rules to calculate the value of the temporal query as we proceed in time (rather than evaluating the query over the history). Let us consider a simple example. We will use the presence of the rule name $r_{p \mathcal{S}^{-} q}$ in the rule activation state to denote that the pure past temporal formula $p \mathcal{S}^{-} q$ holds at that moment in the evaluation. We then use a rule, named $r_{p: p \mathcal{S}^{-} q \text { ? }}$, to calculate whether $r_{p \mathcal{S}^{-}}$should be made active because $p$ held in the previous moment (similarly for the other possible way for $p \mathcal{S}^{-} q$ to hold). These query rules must be universally active in order to determine truth values for the next moment. Thus we use a rule, named say $r_{g . p \mathcal{S}^{-}} q$ ? , to act as a generator for the set of rules that determine the truth of $p \mathcal{S}^{-} q$ based on the previous values of its subformulas.

$$
\begin{aligned}
& r_{q . p \mathcal{S}^{-} q ?}: q \rightarrow r_{p \mathcal{S}^{-} q} \\
& r_{p . p \mathcal{S}^{-} q ?}: p, r_{p \mathcal{S}^{-} q} \longrightarrow r_{p \mathcal{S}^{-} q} \\
& r_{g . p \mathcal{S}^{-} q ?}: \multimap r_{g . p \mathcal{S}^{-} q ?}, r_{p . p \mathcal{S}^{-} q ?}, r_{q . p \mathcal{S}^{-} q \text { ? }}
\end{aligned}
$$

Naturally, the above translation scheme must be generalized to take into account that the subformulas of $\psi \mathcal{S}^{-} \phi$ may be Boolean combinations of pure past-time temporal formulas (represented by rule names) and/or literals. Let $W F F^{-}$denote the set of pure past temporal formulas and $W F F^{-0}$ the set of present and pure past-time temporal formulas. We thus define a translation $\overleftarrow{T}$ that will translate a 
past-time temporal formula (from $W F F^{-0}$ ) into an extension of a rule system $\langle R, O, P, I, S, Q\rangle$ where the fields $R, O, P$ and $I$ are as defined in a rule system, $S$ denotes a set of initial starting values ${ }^{7}$ for the rules representing 'since' ('zince') formulas and $Q$ is a set of rule names denoting the past-time queries calculated by the rule system. For example, a formula $\phi \mathcal{S}^{-} \psi$ must be false initially and so we include the negated rule name $\neg r_{\phi \mathcal{S}^{-} \psi}$ in the set $S$, on the other hand, a formula $\phi \mathcal{Z}^{-} \psi$ is evaluated as true at the beginning of time and therefore we include the associated rule name $r_{\phi \mathcal{Z}^{-}} \psi$ in the set $S$. For a Boolean combination of past-time formulas, such as $p \mathcal{S}^{-} q \wedge\left(r \mathcal{Z}^{-} s \vee p \mathcal{S}^{-} r\right)$, the set $Q$ will be $\left\{\left\{r_{p \mathcal{S}^{-}}, r_{r \mathcal{Z}^{-}}\right\},\left\{r_{p \mathcal{S}^{-} q}, r_{p \mathcal{S}^{-} r}\right\}\right\}$.

Figure 4 gives the translation for the present and pure past-time temporal formulas. Note that the key difference between the translation of the $\mathcal{S}^{-}$and $\mathcal{Z}^{-}$temporal connectives is in the definition of the $S$ component. The correctness of this translation is given by the following theorem.

\section{THEOREM 17}

For any NNF formula $\phi \in W F F^{-0}$, observation trace $\tau$ and $i \in 1 . .|\tau|, \tau, i \models \phi$ if and only there is an accepting run $\gamma$ on observation trace $\tau[1 . . i]$ via the rules of $\overleftarrow{T}(\phi)$ and for some $s \in Q(\overleftarrow{T}(\phi))$ we have $s \subseteq \mathcal{A}\left(\gamma_{i}\right) \cup \Theta\left(\gamma_{i}\right)$

Proof. The proof proceeds by induction over the structure of the formula $\phi \in W F F^{-0}$. The interesting subcases are for the temporal operators, each requiring a further induction proof over the index $i$. We consider just the case for $\phi=X \mathcal{S}^{-} Y$. The argument for $X \mathcal{Z}^{-} Y$ is similar.

Base case: This is when $i=1$. Note that $\tau, 1 \not \models X \mathcal{S}^{-} Y$ as there is no past. $Q\left(\overleftarrow{T}\left(X \mathcal{S}^{-} Y\right)\right)=\left\{\left\{r_{X \mathcal{S}^{-} Y}\right\}\right\}$ However, the rule $r_{X \mathcal{S}^{-} Y}$ can not exist in any resultant state of the frontier at index 1 since it is required by the $S$ initial frontier set that $\neg r_{X \mathcal{S}^{-} Y}$ is present. Similarly for the converse. Thus there is no accepting run of $\overleftarrow{T}\left(X \mathcal{S}^{-} Y\right)$ on $\tau[1 . .1]$

Inductive Step: Here we assume the result at index $i<|\tau|$ and show it holds for $i+1$. We make use, of course, of the result holding for the sub-formulas $X$ and $Y$ at any index $i$ (the hypothesis of the outer-level structural induction proof). We also assume, wlog, that the rule names of the systems for the translations of $X$ and $Y$ are disjoint. The definition given in Figure 4 gives three forms of rules for the translation of $X \mathcal{S}^{-} Y$ over and above the rules obtained for $X$ and $Y$. The first rule, the generator, ensures that the second and third forms of rules are always active. Let us consider the 'if' direction of the theorem. We have $\tau, i \models X \mathcal{S}^{-} Y$. By the semantics of the temporal operator $\mathcal{S}^{-}, \tau, i+1 \models X \mathcal{S}^{-} Y$ if and only if (i) $\tau, i \models Y$ or (ii) $\tau, i=X$ and $\tau, i \models X \mathcal{S}^{-} Y$. For subcase (i), the structural inductive hypothesis gives that there is an accepting run of $\overleftarrow{T}(X)$ on $\tau[1 . . i]$ where for some $x \in Q(\overleftarrow{T}(X))$ we have $x \subseteq \mathcal{A}\left(\gamma_{i}\right) \cup \Theta\left(\gamma_{i}\right) \cup \tau_{i}$. Hence by the second rule form we will have that $r_{X \mathcal{S}^{-} Y}$ will be present in $\mathcal{A}\left(\gamma_{i+1}\right) \cup \Theta\left(\gamma_{i+1}\right)$. Alternatively, for subcase (ii), the hypotheses will mean that for some $x \in Q(\overleftarrow{T}(X))$ we have both $x$ and $r_{X} \mathcal{S}^{-} Y$ present in $x \subseteq \mathcal{A}\left(\gamma_{i}\right) \cup \Theta\left(\gamma_{i}\right) \cup \tau_{i}$. The third rule thus ensures that $r_{X \mathcal{S}^{-} Y}$ is present in $\mathcal{A}\left(\gamma_{i+1}\right) \cup \Theta\left(\gamma_{i+1}\right)$. Thus we conclude that there will be an accepting run on $\tau[1 . . i+1]$. The converse follows similar argumentation.

Separated temporal implicative forms: We now bring together the above two translations $\vec{T}$ and $\overleftarrow{T}$ to define a translation $T$ for the METATEM-like rule form $\phi_{\text {past }} \Rightarrow \bigcirc \psi_{\text {future }}$ which is of universal nature,

\footnotetext{
${ }^{7}$ We distinguish this set $S$ from $I$ as $I$ may also contain initial values of observation propositions. $S$, in a sense, is the past-time counterpart to the forbidden set $F$ in a rule system proper.
} 


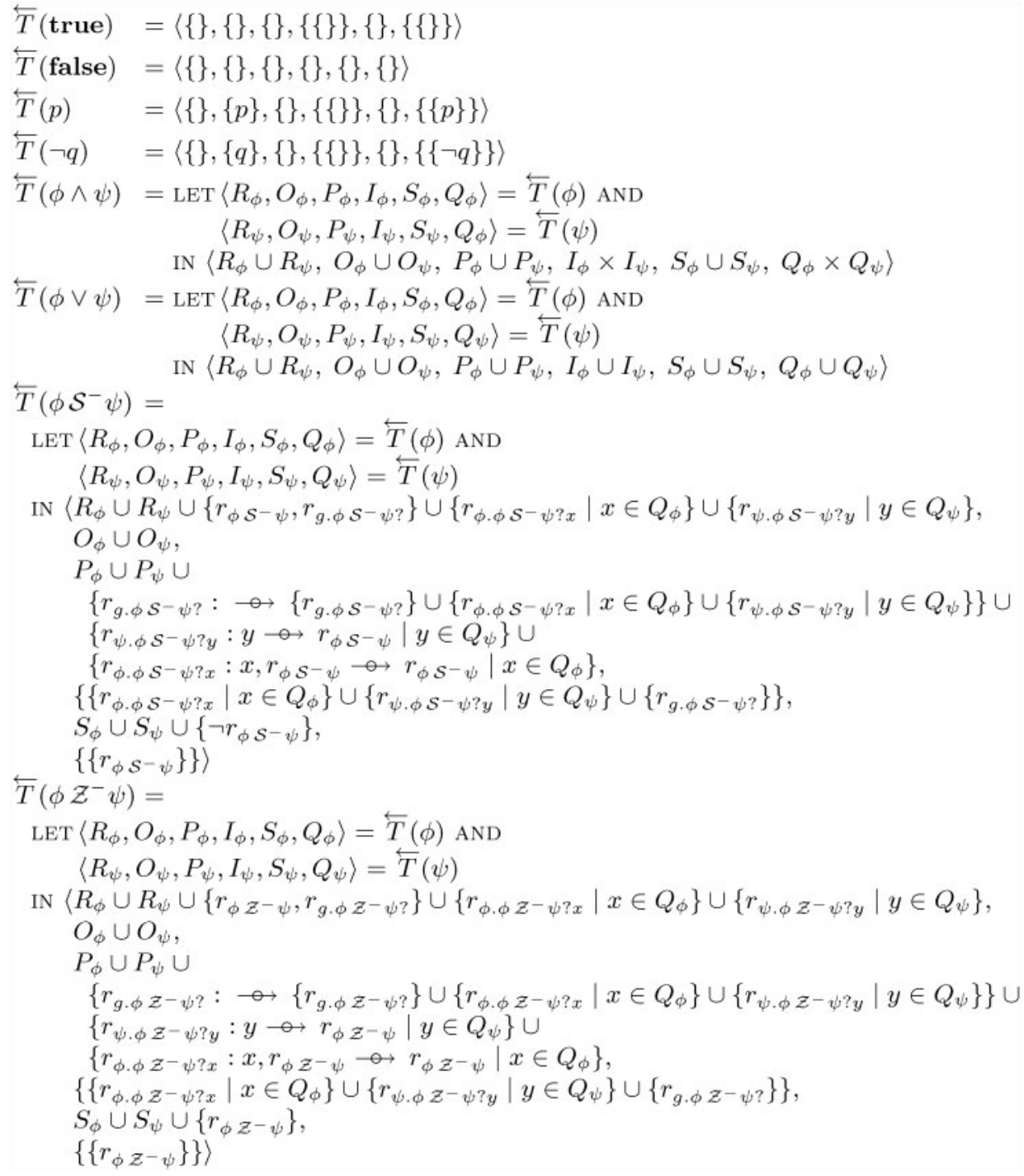

FIGURE 4. Translation of present and past-time temporal query formulas

i.e. globally holds. A translation scheme is given in Figure 5. The translation of the present- and/or past-time formula $\phi$ will yield a set of sets of rule names that represent the query. Presence of one of the sets of rule instances at some stage during the evaluation denotes the truth of the $\phi$ at that point. The translation of the future-time formula $\psi$ will provide a set of sets of rule instances/observations (the initial frontier) such that a successful run starting with that initial frontier over an observation trace will mean the observation trace will satisfy the formula. Thus, the translation for the separated implicative rule needs to create a set of rules such that each element of $Q(\overleftarrow{T}(\phi))$ is combined as an antecedent with the initial frontier $I(\vec{T}(\psi))$ as consequent. Each such constructed RULER rule then needs to be made global. 


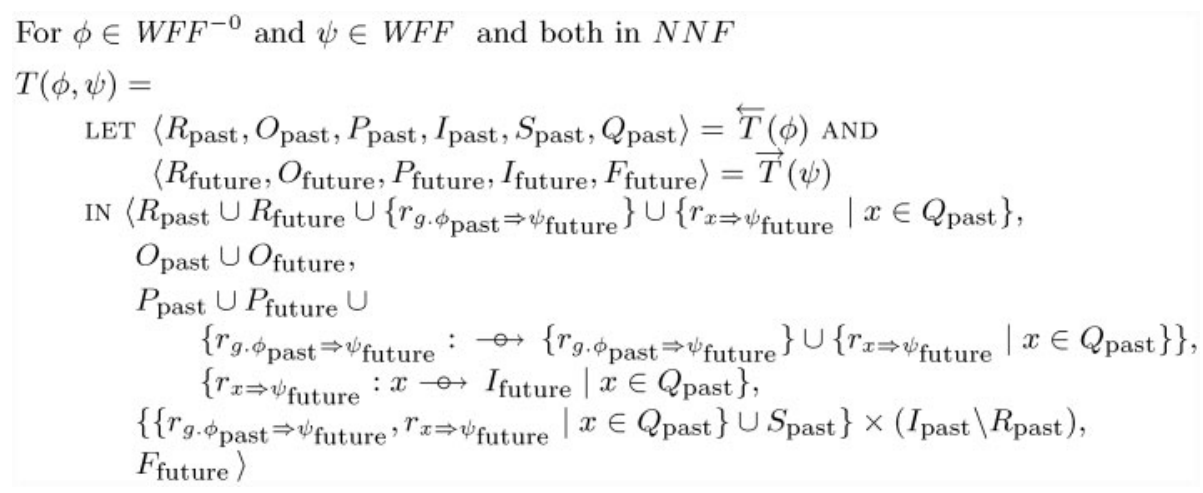

FIGURE 5. Translation of global temporally separated implicative rules

EXAMPLE 18

Let $a, b, c, p$ and $q$ denote propositions. We give the RULER translation of the universal separated temporal implication

$$
c \wedge\left(b \mathcal{S}^{-} a\right) \Rightarrow \bigcirc(\diamond p \wedge \diamond q) .
$$

Recall that $\diamond p$ will be translated as $p \vee \oplus p$, i.e. $p \vee$ true $\mathcal{U}^{+} p$, similarly for $\diamond q$. Using the following abbreviations

$$
\begin{aligned}
& r_{0}=r_{g . b \mathcal{S}^{-}} a ? \quad r_{1}=r_{b \mathcal{S}^{-} a ? b} \quad r_{2}=r_{b \mathcal{S}^{-}} a ? a \quad r_{3}=r_{b \mathcal{S}^{-} a}
\end{aligned}
$$

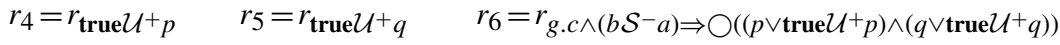

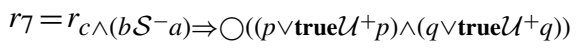

the translation yields the rule system with rules

$$
\begin{array}{lll}
r_{0}: \multimap r_{0}, r_{1}, r_{2} & r_{1}: b, r_{3} \rightarrow r_{3} & r_{2}: a \multimap r_{3} \\
r_{3}: & r_{4}: \multimap p \mid r_{4} & r_{5}: \multimap q \mid r_{5} \\
r_{6}: \multimap r_{6}, r_{7} & r_{7}: c, r_{3} \rightarrow p, q\left|p, r_{5}\right| r_{4}, q \mid r_{4}, r_{5}
\end{array}
$$

and an initial rule activation set $\left\{\left\{\neg r_{3}, r_{0}, r_{1}, r_{2}, r_{6}, r_{7}\right\}\right\}$ and the forbidden rule set $\left\{r_{4}, r_{5}\right\}$.

\section{Theorem 19}

For any (finite) observation trace $\tau$, present- or past-time formula $\phi \in W F F^{-0}$ and future time formula $\psi \in W F F$ we have $\tau, 0=\square(\phi \Rightarrow \bigcirc \psi)$ if and only if there is an accepting run of $T(\phi, \psi)$ for the observation trace $\tau$.

Proof. From the definition of the temporal operator $\square$, we need to establish that for all $i \in 1 . .|\tau|$ $\tau, i \models \phi \Rightarrow \bigcirc \psi$ if and only if there is an accepting run of $T(\phi, \psi)$ for the observation trace $\tau$.

if: we assume that for any $i$ if $\tau, i \models \phi$ then $\tau, i+1 \models \psi$. We show that there is an accepting run of $T(\phi, \psi)$ on $\tau$. Wlog, we assume that $\overleftarrow{T}(\phi)$ and $\vec{T}(\psi)$ have distinct rule names. Given $\tau, i \models \phi$, by Theorem 17 we have that there is an accepting run $\gamma$ over $\tau[1 . . i]$ for which some set $x \in Q(\overleftarrow{T}(\phi))$ is a subset of one of the resultant states at index $i$. The translation $T(\phi, \psi)$ generates a rule $r_{x \Rightarrow \psi}: x \rightarrow I(\vec{T}(\psi))$ for each $x \in Q(\overleftarrow{T}(\phi))$ which is made global, i.e. active for every step. The rule system frontier at $i+1$ thus features $I(\vec{T}(\psi))$. But by Theorem 16, $\tau, i+1 \models \psi$ if and only there is an accepting run of $\vec{T}(\psi)$ on $\tau^{(i+1)}$. As $T(\phi, \psi)$ contains all the rules of $\vec{T}(\psi)$ and $I \overrightarrow{(T}(\psi))$ features in the frontier at step $i+1$, we thus have that there'll be an accepting run of $T(\phi, \psi)$ over $\tau$. 
only if: for this case, we show that under the assumption that for some $i$ we have $\tau, i \models \phi$ and $\tau, i+1 \not \models \psi$ then there is not an accepting run of $T(\phi, \psi)$ over $\tau$. By Theorems 17 and 16 we have, respectively, there is an accepting run of $\overleftarrow{T}(\phi)$ on $\tau[1 . . i]$ but not an accepting run of $\vec{T}(\psi)$ on $\tau^{(i+1)}$. The rule system for $T(\phi, \psi)$ thus has a run over $\tau[1 . . i]$ for which some set $x \in Q(\overleftarrow{T}(\phi))$ is a subset of one of the resultant states at index $i$. The globally active rule $r_{x \Rightarrow \psi}: x \rightarrow I(\vec{T}(\psi))$ for each $x \in Q(\overleftarrow{T}(\phi))$ thus embeds $I(\vec{T}(\psi))$ across the frontier at $i+1$ Now given that there is no accepting run of $\vec{T}(\psi)$ on $\tau^{(i+1)}$ and the rules of $\vec{T}(\psi)$ are a part of $T(\phi, \psi)$, there will be no accepting run over $\tau$.

Finally, in order to obtain a translation for an arbitrary linear-time temporal logic formula, one needs to translate the given formula to the above separated implicative temporal rule forms together with any initial constraints. The translation is detailed but can be generated from the algorithm for separation outlined in Ref. [12]. As was argued with METATEM, however, it is often more natural to write temporal properties directly in this particular separated implicative form.

\section{Parameterized RuleR}

The power and flexibility of rule-based systems arises, in part, through rule parametrization. We therefore consider, in this section, a version of RULER in which (i) rule names can be parameterized by both rule expressions and by data, (ii) observations may be represented by predicates applied to ground terms and (iii) Boolean combinations of relations over arithmetic terms are allowed in antecedents and consequents of the rule bodies.

\subsection{RULER with rule-expression arguments}

We first consider an extension of RULER to include rule definitions parameterized by rule expressions. The propositional RULER system corresponds to regular languages, which are a subclass of propositional EAGLE. This first, seemingly small, extension increases the formal expressivity of RULER to beyond context-free languages, as we show through the combination of the general result of Section 6.2 and the specific case of Example 21.

We introduce arguments to rule definitions in an obvious way. In the rule definition below, $\rho$ is a formal argument to rule $r$. A formal rule argument may then stand in a rule literal in the condition or body part of the rule, or appear as an actual argument to a rule.

$$
r(\rho): a \multimap b, \rho \mid c, r(\rho)
$$

Let us now consider the use of rule $r$ in the monitoring algorithm. Consider a frontier comprising one rule activation state, in turn comprising two different activations of rule $r$, namely

$$
\left\{\left\{r\left(r_{0}\right), r\left(r\left(r_{0}\right)\right)\right\}\right\}
$$

together with an observation state $\{a\}$. The rule activation $r\left(r_{0}\right)$ binds the formal argument $\rho$ of the rule definition $r$ to the rule expression $r_{0}$. Hence, application of the instantiated rule schema will contribute the obligations

$$
\left\{\left\{b, r_{0}\right\},\left\{c, r\left(r_{0}\right)\right\}\right\}
$$


to the next frontier. The rule activation $r\left(r\left(r_{0}\right)\right)$, on the other hand, creates a different binding for $\rho$, i.e. to $r\left(r_{0}\right)$ and hence will contribute obligations

$$
\left\{\left\{b, r\left(r_{0}\right)\right\},\left\{c, r\left(r\left(r_{0}\right)\right)\right\}\right\}
$$

to the frontier. These are the only two rule activations, hence, the next frontier is the (consistent) product of the two generated obligation frontiers, i.e.

$$
\left\{\left\{b, r_{0}, r\left(r_{0}\right)\right\},\left\{b, r_{0}, c, r\left(r\left(r_{0}\right)\right)\right\},\left\{c, r\left(r_{0}\right), b\right\},\left\{c, r\left(r_{0}\right), r\left(r\left(r_{0}\right)\right)\right\}\right\} .
$$

The monitoring, or evaluation, algorithm for this rule-expression parameterized version of RULER follows the basic high-level steps of the algorithm informally presented in Section 2. The key differences, from an implementation point of view, are that (i) rule activations are now rule term structures rather than just rule names and that (ii) bindings of formal rule argument names to actual rule term structures must be created and used to apply rule schema in the construction of the successor frontier.

We give further demonstration of the monitoring process using two small examples that show how RULER, when parameterized by just rule-expressions, can define context-free and context-sensitive languages.

\section{EXAMPLE 20}

Let us consider the classic context-free language $\left\{a^{n} b^{n} \mid n \geq 1\right\}$. We encode it in RULER as a rule system accepting the language $\left\{\{a, \neg b\}^{n}\{\neg a, b\}^{n} \mid n \geq 1\right\}$ where $a$ and $b$ are observation propositions. We use the rule schema

$$
\begin{array}{ll}
r_{b}(\rho): \multimap b, \neg a, \rho & r_{a b}(\rho): \multimap b, \neg a, \rho \mid a, \neg b, r_{a b}\left(r_{b}(\rho)\right) \\
r_{\text {end }}: \multimap r_{\text {fail }} & r_{\text {fail }}: \multimap r_{\text {fail }}
\end{array}
$$

together with its initial rule activation set as $\left\{\left\{a, r_{a b}\left(r_{\text {end }}\right)\right\}\right\}$ and its final forbidden rule set as $\left\{r_{b}, r_{a b}, r_{f a i l}\right\}$ (meaning that no occurrence of rule $r_{b}, r_{a b}$, nor $r_{\text {fail }}$, may appear as an obligation in a final rule activation state). Below in Table 2 we spell out the evaluation over an input trace corresponding to three $a$ s followed by three $b$ s (we assume only one of $a$ or $b$ may be true at any one time).

\begin{tabular}{|c|c|c|c|}
\hline Step & Obs. & Rule activations & Resultant states \\
\hline 0 & $\{a, \neg b\}$ & $\left\{\left\{a, r_{a b}\left(r_{e n d}\right)\right\}\right\}$ & $\left\{\left\{a, \neg b, r_{a b}\left(r_{e n d}\right)\right\}\right\}$ \\
\hline 1 & $\{a, \neg b\}$ & $\left\{\begin{array}{l}\left\{b, \neg a, r_{\text {end }}\right\}, \\
\left\{a, \neg b, r_{a b}\left(r_{b}\left(r_{\text {end }}\right)\right)\right\}\end{array}\right\}$ & $\left\{\left\{a, \neg b, r_{a b}\left(r_{b}\left(r_{e n d}\right)\right)\right\}\right\}$ \\
\hline 2 & $\{a, \neg b\}$ & $\left\{\begin{array}{l}\left\{b, \neg a, r_{b}\left(r_{\text {end }}\right)\right\} \\
\left\{a, \neg b, r_{a b}\left(r_{b}\left(r_{b}\left(r_{\text {end }}\right)\right)\right)\right\}\end{array}\right\}$ & $\left\{\left\{a, \neg b, r_{a b}\left(r_{b}\left(r_{b}\left(r_{\text {end }}\right)\right)\right)\right\}\right\}$ \\
\hline 3 & $\{\neg a, b\}$ & $\left\{\begin{array}{l}\left\{b, \neg a, r_{b}\left(r_{b}\left(r_{\text {end }}\right)\right)\right\} \\
\left\{a, \neg b, r_{a b}\left(r_{b}\left(r_{b}\left(r_{b}\left(r_{\text {end }}\right)\right)\right)\right)\right\}\end{array}\right\}$ & $\left\{\left\{\neg a, b, r_{b}\left(r_{b}\left(r_{\text {end }}\right)\right)\right\}\right\}$ \\
\hline 4 & $\{\neg a, b\}$ & $\left\{\left\{b, \neg a, r_{b}\left(r_{\text {end }}\right)\right\}\right\}$ & $\left\{\left\{\neg a, b, r_{b}\left(r_{\text {end }}\right)\right\}\right\}$ \\
\hline 5 & $\{\neg a, b\}$ & $\left\{\left\{b, \neg a, r_{\text {end }}\right\}\right\}$ & $\left\{\left\{\neg a, b, r_{\text {end }}\right\}\right\}$ \\
\hline
\end{tabular}

Let us look at the situations in step 2 and step 3. The resultant state in step 2 activates rule $r_{a b}$ with argument $r_{b}\left(r_{b}\left(r_{\text {end }}\right)\right)$ denoting that if $b$ occurs next in step 3 then two further $b$ s will be required to

TABLE 2. Example evaluation on $a a a b b b$ 
TABLE 3. Evaluation trace on $a a a b b b a b$

\begin{tabular}{llll}
\hline Step & Obs. & Rule activations & Resultant states \\
\hline 5 & $\{\neg a, b\}$ & $\left\{\left\{b, \neg a, r_{\text {end }}\right\}\right\}$ & $\left\{\left\{\neg a, b, r_{\text {end }}\right\}\right\}$ \\
6 & $\{a, \neg b\}$ & $\left\{\left\{r_{\text {fail }}\right\}\right\}$ & $\left\{\left\{a, \neg b, r_{\text {fail }}\right\}\right\}$ \\
7 & $\{\neg a, b\}$ & $\left\{\left\{r_{\text {fail }}\right\}\right\}$ & $\left\{\left\{\neg, b, r_{\text {fail }}\right\}\right\}$ \\
\hline
\end{tabular}

TABLE 4. Evaluation trace on $a a a b b a$

\begin{tabular}{llll}
\hline Step & Obs. & Rule activations & Resultant states \\
\hline 4 & $\{\neg a, b\}$ & $\left\{\left\{b, \neg a, r_{b}\left(r_{\text {end }}\right)\right\}\right\}$ & $\left\{\left\{\neg a, b, r_{b}\left(r_{\text {end }}\right)\right\}\right\}$ \\
5 & $\{a, \neg b\}$ & $\left\{\left\{b, \neg a, r_{\text {end }}\right\}\right\}$ & \{\} \\
\hline
\end{tabular}

match the $a$ s that have been seen so far. Expansion of the particular rule call gives two possible rule activation sets in step 3 , the second of which is now inconsistent with the given observations $\{\neg a, b\}$. Hence the resultant state with just rule $r_{b}$ active [with argument $r_{b}\left(r_{\text {end }}\right)$ ]. By step 5, the evaluation of the rule system over the trace is successful-the final (resultant) state has no forbidden rules.

Suppose, now, the input had not terminated and continued with two more observations states. The evaluation would proceed from step 5 as in Table 3. The 'illegal' input (appearing in steps 6 and 7 ) is consumed, however, the rule $r_{\text {fail }}$ is activated through $r_{\text {end }}$ in the merged state of step 5 and maintained active until the input is completed. The evaluation then fails since the rule $r_{\text {fail }}$ is one of the forbidden rules. Terminating earlier than expected results in either $r_{a b}$ or $r_{b}$ present in the final (merged) state, which are also forbidden rules. Alternatively, if the input has too few $b$ s and then continues with $a$ s, the frontier of merged states becomes vacuous which then causes the rule system to stop and fail to accept the input, as shown in the alternative continuation from step 4 in Table 4 . In fact, all accepted observation traces will match against a trace of $n \geq 1$ occurrences of $a$ followed by $n$ occurrences of $b$. Essentially, barring the first $a$, the rule $r_{a b}$ represents the non-terminal $S$ of the context-free grammar with production rule $S=a b \mid a S b$ in which $r_{a b}$ 's actual argument represents the continuation string for concatenation to the string of $a$ 's generated.

Note, however, that the space requirements for each successive evaluation grows linearly while $a$ s are being observed; this is, of course, a heavy penalty for monitoring, but it is the cost for using just rule-expression parameters. Example 24 gives a constant space rule system encoding.

EXAMPLE 21

Here we encode the context-sensitive language $\left\{a^{n} b^{n} c^{n} \mid n \geq 1\right\}$. Let us first extend the previous example to accept traces of the form

$$
\{a, \neg b, \neg c\}^{n}\{\neg a, b, \neg c\}^{n}\{\neg a, \neg b, c\}^{m} \text {, for } n, m \geq 1 .
$$

We use the rule set

$$
\begin{aligned}
& r_{a b}(\rho): \multimap b, \neg a, \neg c, \rho \mid a, \neg b, \neg c, r_{a b}\left(r_{b}(\rho)\right) \\
& r_{b}(\rho): \multimap \rightarrow b, \neg a, \neg c, \rho \\
& r_{c}: \multimap c, \neg a, \neg b, r_{\text {end }} \mid c, \neg a, \neg b, r_{c} \\
& r_{\text {end }}: \multimap r_{\text {fail }} \\
& r_{\text {fail }}: \multimap r_{\text {fail }}
\end{aligned}
$$


together with an initial activation set defined as $\left\{\left\{a, r_{a b}\left(r_{c}\right)\right\}\right\}$ and the final forbidden rule activation set defined as $\left\{r_{a b}, r_{b}, r_{c}, r_{f a i l}\right\}$. This system will clearly accept traces of the form $a^{n} b^{n}$ (represented by the $r_{a b}$ rule) followed by one or more $c$ 's (determined by the $r_{c}$ argument to the initial rule activation $\left.r_{a b}\right)$. In a similar way, we can encode the language $a^{m} b^{n} c^{n}$ for $n, m \geq 1$. Use the rules

$$
\begin{aligned}
& r_{a}(\rho): \multimap b, \neg a, \neg c, \rho \mid a, \neg b, \neg c, r_{a}(\rho) \\
& r_{b c}(\rho): \multimap c, \neg a, \neg b, \rho \mid b, \neg a, \neg c, r_{b c}\left(r_{c 1}(\rho)\right) \\
& r_{c 1}(\rho): \multimap c, \neg a, \neg b, \rho \\
& r_{\text {end }}: \multimap r_{\text {fail }} \\
& r_{\text {fail }}: \multimap \rightarrow r_{\text {fail }}
\end{aligned}
$$

with an initial frontier set $\left\{\left\{a, r_{a}\left(r_{b c}\left(r_{e n d}\right)\right\}\right\}\right.$ and forbidden rule activation set $\left\{r_{a}, r_{b c}, r_{c 1}, r_{f a i l}\right\}$.

Now we can encode the intersection of the rule systems accepting languages, $a^{n} b^{n} c^{m}$ and $a^{m} b^{n} c^{n}$ ( $n, m \geq 1)$, to yield a rule system accepting the context-sensitive language $a^{n} b^{n} c^{n 8}(n \geq 1)$. This is given as the union of the above rule sets and product of the initial frontier sets and union of the forbidden rule activation set.

So, the product rule system has an initial activation set $\left\{\left\{a, r_{a b}\left(r_{c}\right), r_{a}\left(r_{b c}\left(r_{e n d}\right)\right)\right\}\right\}$ and final forbidden rule activation set $\left\{r_{a b}, r_{b}, r_{c}, r_{a}, r_{b c}, r_{c 1}, r_{f a i l}\right\}$.

\subsection{Context-free grammars in RULER}

Example 20 suggests a general result.

THEOREM 22

The class of context-free languages is a strict subset of those accepted by rule-parameterized RULER.

We outline the proof of this result by defining a translation of context-free grammars into RULER systems. The example encoding of the language defined by $a^{n} b^{n} c^{n}$, in Example 21, then establishes the strict inclusion.

We start by noting that any context-free grammar can be transformed into Greibach Normal Form [14], whose production rules are either

- of the form $N \rightarrow T N_{1} N_{2} \ldots N_{n}(n \geq 0)$, for a terminal symbol $T$ and non-terminal symbols $N$ with the starting symbol $S$ not appearing on the right-hand side, or

- $S \rightarrow \lambda$, for starting symbol $S$ and empty word $\lambda$.

Thus, consider a grammar $G$ in Greibach Normal Form over terminal alphabet $\Sigma$. First, we convert production rules of the form $N \rightarrow T N_{1} N_{2} \ldots N_{n}(n \geq 0)$ into parameterized right recursive rules as follows:

1. convert rules of the form $N \rightarrow T$, where $T$ is a terminal symbol and $N$ is not the start non-terminal $S$, into parameterized rule $N(x) \rightarrow T x$;

2. convert rules of the form $N \rightarrow T N_{1} N_{2} \ldots N_{n}(n>0)$, where $N$ is not the start non-terminal $S$, into

$$
N(x) \rightarrow T N_{1}\left(\ldots\left(N_{n}(x)\right) \ldots\right)
$$

\footnotetext{
${ }^{8}$ represented, of course, as the set of traces $\{a, \neg b, \neg c\}^{n}\{\neg a, b, \neg c\}^{n}\{\neg a, \neg b, c\}^{n}$.
} 
3. convert rules of the form $S \rightarrow T$, where $T$ is a terminal symbol, into the rule $S \rightarrow T E$ where $E$ is a special non-terminal introduced for an empty production;

4. convert rules of the form $S \rightarrow T N_{1} N_{2} \ldots N_{n}(n>0)$, for start non-terminal symbol $S$, into rules of the form

$$
S \rightarrow T N_{1}\left(\ldots\left(N_{n}(E)\right) \ldots\right)
$$

We then generate a RULER rule $r_{N}$ for each non-terminal symbol $N$. Consider the set of parameterized production rules for an arbitrary non-start non-terminal symbol $N$. We generate one RULER rule for the whole set, which is of the form

$$
r_{N}(x): \multimap \ldots\left|\widehat{T}_{i}, r_{N_{1}^{i}}\left(\ldots\left(r_{N_{n_{i}}^{i}}(x)\right) \ldots\right)\right| \ldots
$$

where each of the disjuncts corresponds to the right-hand side of a rule in the set, and viceversa. For a terminal symbol $T_{0}$, we use $\widehat{T}_{0}$ to denote the (conjunctive) list of RULER observations $T_{0}, \neg T_{1}, \neg T_{2}, \ldots, \neg T_{|\Sigma|-1}$ for $T_{i} \in \Sigma$ and $T_{i}=T_{j}$ if and only if $i=j$. Note that for a rule with right-hand side $T x$, with terminal symbol $T$ and parameter $x$, the corresponding disjunct is $\widehat{T}, x$. Similarly, the non-empty start symbol rules generate the RULER rule

$$
r_{S}: \multimap \ldots\left|\widehat{T}_{i}, r_{N_{1}^{i}}\left(\ldots\left(r_{N_{n_{i}}^{i}}\left(r_{E}\right)\right) \ldots\right)\right| \ldots
$$

The RULER rule for $r_{E}$ is simply $r_{E}: \multimap$ and is used as a terminator. If the original grammar has the production $S \rightarrow \lambda$, then the initial set, i.e. frontier, for the rule system is $\left\{r_{S} \mid r_{E}\right\}$ otherwise it is just $\left\{r_{S}\right\}$. The forbidden set is the set of all rule names apart from $r_{E}$.

We claim that the rule system so generated accepts the language $\{t w \mid t \in \Sigma, w \in \mathcal{L}(G)\}$. The remaining task is to apply a transformation to the rule set to shift the words accepted one place left so as to lose the arbitrary first symbol $t$. We demonstrate such a transformation on a small example rather than describe the detailed steps, which are notationally awkward.

A Greibach Normal Form for the simple grammar $S \rightarrow a S b$ and $S \rightarrow \lambda$ is:

$$
\begin{aligned}
& S \rightarrow \lambda|a B| a A B \\
& A \rightarrow a B \mid a A B \\
& B \rightarrow b
\end{aligned}
$$

Using the above translation, the RULER rules generated from this grammar are:

$$
\begin{aligned}
& r_{S}: \multimap a, \neg b, r_{B}\left(r_{E}\right) \mid a, \neg b, r_{A}\left(r_{B}\left(r_{E}\right)\right) \\
& r_{A}(x): \multimap a, \neg b, r_{B}(x) \mid a, \neg b, r_{A}\left(r_{B}(x)\right) \\
& r_{B}(x): \multimap b, \neg a, x \\
& r_{E}: \multimap \rightarrow
\end{aligned}
$$

The idea of the shift transformation is to move the terminal symbol constraint appearing in the consequent of a rule $r$ into the rules that may 'call' the rule $r$. Thus for each parameterized rule $r_{N}(x)$, we introduce rules $r_{N t}(x)$ for each terminal symbol $t$ that may be the first symbol accepted by the rule argument $x$. We name the revised starting rule as $r_{S}{ }^{\prime}$ and replace by $r_{E}$ those occurrences of rule 
applications $r_{N}\left(r_{E}\right)$ appearing in the right-hand sides of the start rule $r_{S}$. For the above example, the transformed rules are then as follows.

$$
\begin{aligned}
& r_{S}^{\prime}: \multimap b, \neg a, r_{E} \mid a, \neg b, r_{A b}\left(r_{E}\right) \\
& r_{A b}(x): \multimap b, \neg a, r_{B b}(x) \mid a, \neg b, r_{A b}\left(r_{B b}(x)\right) \\
& r_{B b}(x): \multimap b, \neg a, x \\
& r_{E}: \multimap \rightarrow
\end{aligned}
$$

The initial frontier of the rule system then becomes $\left\{a, \neg b, r_{S}^{\prime} \mid r_{E}\right\}$.

\subsection{Including data parameters in RULER}

As in EAGLE, we can also parameterize RULER rules by data values, thus introducing variables and predicated atoms. It is through such means that RULER can be used for encoding/interpreting realtime and stochastic logics, as well as enabling encodings of monitoring formulas that can be more efficiently evaluated. As was the case for the inclusion of rule-expression arguments, the basic highlevel steps of the monitoring algorithm are not effected by this extension. The binding mechanism used for rule-expressions handles data expressions as well. We therefore just exemplify the concepts with two further examples.

\section{EXAMPLE 23}

Let us assume that each observation state is 'time-stamped' by the inclusion of a unique grounded predicate $\operatorname{clock}(t)$ for some real value $t$, e.g. one might have an observation state $\{p, \operatorname{clock}(49738.22264)\}$ indicating that $p$ occurred at time 49738.22264. The data parameterized rule schema

$$
\begin{aligned}
r(k: \mathbb{R}): \operatorname{clock}(n: \mathbb{R}) \rightarrow & \operatorname{clock}(t: \mathbb{R}), p, t-n<k \mid \\
& \operatorname{clock}(t: \mathbb{R}), \neg p, t-n<k, r(k-t+n)
\end{aligned}
$$

defines a constraint that the atom $p$ must be consistent with an observation state within $k$ time units from the observation state in which the rule $r(k)$ is required to hold. The $n: \mathbb{R}$ appearing as argument to the clock predicate name in the rule's condition means that the variable $n$ is to be bound to some value from $\mathbb{R}$ by the current observation state. The occurrence of $\operatorname{clock}(t: \mathbb{R})$ in the rule consequent means that there is an obligation on the next observation state to binding $t$ with some value. Suppose we have an observation state containing just $\{\operatorname{clock}(1), \neg p, r(3)\}$. The rule $r(3)$, through binding $n$ to 3 , gives rise to the set $\{\{\operatorname{clock}(t: \mathbb{R}), p, t-1<3\},\{\operatorname{clock}(t: \mathbb{R}), \neg p, t-1<3, r(4-t)\}\}$. If the next actual observation state is $\{\operatorname{clock}(3), \neg p\}$, the merge with the obligation sets yields the frontier set $\{\{\operatorname{clock}(3), \neg p, r(1)\}\}$, which gives rise, through $r(1)$, to obligations $\{\{\operatorname{clock}(t: \mathbb{R}), p, t-3<$ $1\},\{\operatorname{clock}(t: \mathbb{R}), p, t-3<1, r(4-t)\}\}$. If we have another observation this time with $\{\operatorname{clock}(4), p\}$ then the merge yields the empty frontier set as $4-3<1$, which appears in both possible futures, is clearly false. Hence the actual behaviour does not conform to that required by the initial $r(3)$. On the other hand, had the observation state been, say, $\{\operatorname{clock}(3.9), p\}$, then the rule set would be satisfied.

\section{EXAMPLE 24}

In contrast to the encoding of context-sensitive language $a^{n} b^{n} c^{n}$ for $n>1$ given in Example 21, we provide a simpler and less memory expensive encoding using data parameters. The rule system first counts the number of $a$ s, then attempts to match the observation with $n b$ s followed by $n c s$. Any mismatch on this requirement leads to non-acceptance. The evaluation of the rule system requires constant space for each evaluation step in contrast to the previous given example that requires linear space. Informally, if the rule $r_{a}(n)$ is active then $n$ as have been previously observed; if the rule 
TABLE 5. A nested call return observation trace

\begin{tabular}{|c|c|c|c|c|c|c|c|c|c|c|}
\hline \multicolumn{10}{|c|}{ Observation State Number } \\
\hline 1 & 2 & 3 & 4 & 5 & 6 & 7 & 8 & 9 & 10 & 11 \\
\hline \hline & call & & & & & & & & $\begin{array}{c}\text { return } \\
p\end{array}$ & \\
$b$ & $p$ & & & & & & & & $p$ & \\
\hline & & begin & call & & & & $\begin{array}{c}\text { return } \\
\text { end }\end{array}$ & & \\
& & $q$ & $q$ & & & & $q$ & & \\
\hline & & & & $\begin{array}{c}\text { begin } \\
r\end{array}$ & $r$ & end & & & & \\
& & & & $r$ & & & & \\
\hline
\end{tabular}

$r_{b}(n, m)$ is active then $n$ more $b$ s are required and $m c \mathrm{~s}$; and if $r_{c}(m)$ is active then $m c \mathrm{~s}$ are still required. The rule set is given as below.

$$
\begin{aligned}
& r_{a}(n: \mathbb{N}): \quad \rightarrow a, \neg b, \neg c, r_{a}(n+1) \mid \\
& \neg a, b, \neg c, r_{b}(n-1, n) \\
& r_{b}(n: \mathbb{N}, m: \mathbb{N}): \multimap \neg a, b, \neg c, n>0, r_{b}(n-1, m) \mid \\
& \neg a, \neg b, c, n=0, m>1, r_{c}(m-1) \mid \\
& \neg a, \neg b, c, n=0, m=1, r_{\text {end }} \\
& r_{c}(m: \mathbb{N}): \quad \rightarrow \neg a, \neg b, c, m>1, r_{c}(m-1) \mid \\
& \neg a, \neg b, c, m=1, r_{\text {end }} \\
& r_{\text {end }}: \quad \rightarrow r_{\text {fail }} \\
& r_{\text {fail }}: \quad \rightarrow r_{\text {fail }}
\end{aligned}
$$

The rule system has an initial frontier set given as $\left\{\left\{a, r_{a}(1)\right\}\right\}$ and the forbidden rule set is $\left\{r_{a}, r_{b}, r_{c}, r_{\text {fail }}\right\}$.

\subsection{Using both data and rule parameters in RULER}

In Ref. [1], Alur, Etessami and Madhusudan introduced a temporal logic of nested calls and returns using abstract temporal modalities for skipping over states corresponding to procedure body invocations, e.g. an abstract next-time operator that jumps from a call state to its matching return state. They hint at past-time versions and other modalities. A recent paper of Rosu, Chen and Ball [17] develop specialized run-time monitors for such a past-time version of CaRet, called ptCaRet. For ptCaRet, it is assumed that observation states include, in addition to other monitored information, at most one of the following propositions, call, return, begin and end. It is further assumed that begin-states immediately follow call-states, and end-states immediately precede return-states and that observation traces are prefixes of traces with matched call and return states. In the next example, we show how a uniform and practically efficient encoding of such ptCaRet temporal operators can be given in RULER.

EXAMPLE 25

We first encode the abstract 'previously' temporal operator introduced in Ref. [17]. We assume that each observation state contains at most one of the propositions call, begin, end and return, and their ordering follows the requirements given in Ref. [17]. Table 5 depicts a possible observation trace over properties represented by the propositions $b, p, q$ and $r$. For ease, we have separated out the 
states according to the nesting of call states. Assuming $\widehat{\bigodot}$ denotes the abstract 'previously' temporal operator, then we have that:

(i) $\widehat{\bigodot} p$ is true only at the observation states numbered 3,10 and 11 ;

(ii) $\widehat{\bigodot} q$ is true only at the observation states numbered 4, 5, 8 and 9;

(iii) $\bigodot r$ is true only at the observation states number 6 and 7 .

Assuming a strict abstract 'since' operator defined by (the least solution of)

$$
\widehat{\phi \mathcal{S}^{-}} \psi \equiv \widehat{\bigodot}\left(\psi \vee\left(\phi \wedge \phi \widehat{\mathcal{S}^{-}} \psi\right)\right)
$$

we then have that:

(i) $\widehat{p \mathcal{S}^{-}} b$ is true only at observation states $2,3,10$ and 11 ;

(ii) $(p \vee q) \widehat{\mathcal{S}^{-}} b$ is true only at observation states $2,3,4,5,8,9,10$ and 11 .

In order to model the abstract temporal operators in RULER one needs to determine matching calls and returns. We thus introduce a rule level $(n)$ that will record for each observation state the current call stack nesting level. We also need to determine the value of a given formula at some matching call observation state. We introduce the rule $R M C(n, x)$ that records whether the rule argument $x$ was true on the most recent call at stack level $n$. We will then use the rule $A P(x)$ to encode the truth of abstract previously temporal operator, which is then defined by the following two rules.

$$
\begin{aligned}
& A P g 1(x): \text { end,level }(m: \mathbb{N}), R M C(m-1, x) \rightarrow A P(x) \\
& A P g 2(x): \neg \text { end }, x \rightarrow A P(x)
\end{aligned}
$$

The first rule determines the value of $A P(x)$ for a return observation state. Recall that an end state must immediately precede a return state. Therefore if the end state is currently at stack level $m$ and the rule argument $x$ was true at the matching call state for stack level $m-1$ then $A P(x)$ must hold in the return state (which for RULER means that the rule must be present). On the other hand, if we are currently not at an end state and the rule argument $x$ holds then $A P(x)$ must also hold in the next observation state.

There are three rules to maintain the call stack level counter, level $(n)$.

$$
\begin{aligned}
& \operatorname{Lg} 1: \operatorname{call}, \text { level }(n: \mathbb{N}) \rightarrow \neg \operatorname{level}(n), \text { level }(n+1) \\
& \operatorname{Lg} 2: \text { end,level }(n: \mathbb{N}) \rightarrow \neg \operatorname{level}(n), \text { level }(n-1) \\
& \operatorname{Lg} 3: \neg \operatorname{call}, \neg \text { end,level }(n: \mathbb{N}) \rightarrow \operatorname{level}(n)
\end{aligned}
$$

The rule $R M C(n, x)$ is also defined using three rules.

$$
\begin{aligned}
& R M C g 1(x): \text { return, level }(n: \mathbb{N}) \rightarrow \neg R M C(n, x) \\
& R M C g 2(x): \text { call, level }(n: \mathbb{N}), x \rightarrow R M C(n, x) \\
& R M C g 3(x): R M C(n: \mathbb{N}, x), \text { level }(m: \mathbb{N}), n<m \rightarrow R M C(n, x)
\end{aligned}
$$

The first rule ensures that $\operatorname{RMC}(n, x)$ is switched off for the state following a return state at stack level $n$. The second rule ensures $\operatorname{RMC}(n, x)$ is switched on in the state following a call state at level $n$ in which $x$ is true. The third rule in the above group propagates $R M C(n, x)$ over more deeply nested observation states. 
The above rule schema then needs to be switched on. For example, if it is required to monitor the abstract temporal property $\widehat{\bigodot} p$ we would include the generator rule

$$
\begin{aligned}
r g: \rightarrow \rightarrow & r g, \\
& A P g 1(p), \operatorname{APg} 2(p), \\
& \operatorname{Lg} 1, \operatorname{Lg} 2, \operatorname{Lg} 3, \\
& R M C g 1(p), \operatorname{RMCg} 2(p), \operatorname{RMCg} 3(p)
\end{aligned}
$$

with an initial frontier set $\{\{r g, \operatorname{Lg} 1, \operatorname{Lg} 3, A P g 2(p), R M C g 2(p), \operatorname{level}(0)\}\}$.

The encoding of the abstract 'since' temporal operator can be given in a similar way using the following four generator rules for determining whether the rule name $A S(x, y)$ should be present in a rule activation state.

$$
\begin{aligned}
& A S g 1(x, y): \neg \text { end }, y \rightarrow A S(x, y) \\
& A S g 2(x, y): \text { end,level }(m: \mathbb{N}), R M C(m-1, y) \rightarrow A S(x, y) \\
& A S g 3(x, y): \neg \text { end, } x, A S(x, y) \rightarrow A S(x, y) \\
& A S g 4(x, y): \text { end,level }(m: \mathbb{N}), R M C(m-1, x), R M C(m-1, A S(x, y)) \\
& \quad \rightarrow A S(x, y)
\end{aligned}
$$

Whilst the above has shown the translation for a specific formula, a uniform translation can be based on these schema as was done for the translation of the past-time fragment of LTL into RULER.

\section{Conclusions}

A low-level rule-based system RULER for run-time monitoring has been introduced. An on-thefly trace-checking algorithm, which checks a finite trace of ground observations for conformance against the rules of the RULER specification on a step-by-step basis, was first informally described for the propositional subset of RULER and then illustrated through examples. A formal semantics of the propositional subset of RULER was given and used to establish correctness of a syntacticlevel translation of linear-time temporal logic incorporating both past and future operators into propositional RULER. A key concept in RULER is that the rules have the capability to switch rules on or off as an evaluation of a rule system proceeds over a trace. We refer to such systems as reactive rule systems/grammars/Kripke structures [13]. For regular grammars, the reactivity does not extend expressivity. However, for general context-free grammars, this reactivity extends the expressivity to non-context free grammars. A relationship with alternating automata [11] and state-alternating context-free grammars [16] is clear, however, a more detailed study of reactive grammars and their place in the complexity hierarchy is work in progress, see Ref. [7] for some initial results and examples.

We illustrated the greater expressiveness of the rule-expression parameterized version of RULER by encoding a context-free and a context-sensitive language as RULER rule systems. We then gave a general translation for any context-free grammar, which thus established the strict containment of context-free languages within that version of RULER. In addition to rule-expression parameters, RULER supports rules parameterized by data expressions, just as in EAGLE. Formally, RULER incorporating counting on the natural numbers is able to simulate a Turing machine. However, the inclusion of data is essential for effective run-time monitoring and leads to practically more efficient rule systems. The semantic details are not difficult and RULER adopts an approach similar to that in first-order MetateM [3]. The inclusion of data, of course, enables RulER to be used to encode real-time and stochastic properties. 


\section{Rule Systems for Run-time Monitoring: from EAGLE to RULER}

We have kept the core elements of RULER syntactically small and concise. There are, however, commonly occurring patterns of rules where some additional syntax in RULER can simplify the rule system presentation. One example is to introduce the notion of 'persistent' rule activations in addition to the basic single-step interpretation RULER has for rule activation; this removes the need for the 'generator' rules that have been used throughout most of the examples. It is also quite common to find a collection of rules always being switched on, or off, collectively, for example, a set of rules characterizing some reoccurring state. Hence another syntactic extension is to introduce names referring to such sets of rules and then treating the new name in a similar way to a rule name. As a brief example, suppose one wished to count the number of $p$ events that occur before a $q$ event. In 'core' RULER this can be encoded by the rules

$$
\begin{aligned}
& A p: p, V(x: \mathbb{N}) \rightarrow V(x+1), A p, A q, G \\
& A q: q, V(x: \mathbb{N}) \rightarrow B(x) \\
& G: \neg p, \neg q, V(x: \mathbb{N}) \multimap G, V(x), A p, A q
\end{aligned}
$$

where the initial frontier is $\{G, V(0), A p, A q\}$. The rule $V(x)$ is used to record the current number of $p$ events seen. The rule $G$ is a generator rule that causes the rules $A p, A q$ and $V(x)$ to persist only when $p$ and $q$ events do not occur. In the following syntactic extension, we provide a named collection of rules with such a form of persistence (introduced by the keyword state).

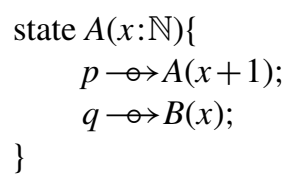

An appropriate initial frontier would now be $\{A(0)\}$ indicating that the count is initially 0 . If $A(n)$ is active and neither of the rules associated with $A(x: \mathbb{N})$ when the formal argument $x$ is replaced by $n$ are 'fired', i.e. the antecedents evaluate false, then $A(n)$ persists to the next monitoring step. If either of the rules do fire, then only the consequents of the associated rules determine the active rules for the next monitoring step. Hence the collective rule $A(x: \mathbb{N})$ is very much like a state in a state-transition system. We have experimented with a range of such syntactic extensions that (i) can be transformed into the 'core' RULER rule forms, (ii) can lead to optimization in the trace-checking algorithm and (iii) can lead to clearer user-level rule system specifications. A tutorial paper on RULER [8] exemplifies many of these extensions that are also implemented in the current Java prototype.

An associated feature not yet fully treated in RULER is rule priority. Given the ability to switch rules on and off, conflicts may occur. Sometimes the conflicts may be desired, but in other situations we may wish one rule to override another, as is the case in handling priority and preferences in default logic (defeasible reasoning) [9]. Of course, this changes the nature of the logics expressible quite considerably and is an area of future development. We note, also, that rule priorities are important for defining transition priority in hierarchical state charts.

The low-level simplicity of RULER leads to the main advantage for its potential use over EAGLE. If optimal (asymptotic) complexity bounds have been established for a particular subset logic of EAGLE, such as for the LTL subset, in general a RULER encoding will be no better asymptotically. However, we assert that smaller constants arise through the significant reduction in the symbolic processing that has to be undertaken at run-time in the interpretation of EAGLE formulas. Of course, there would be a one-off translation cost from the LTL formula to the appropriate rule systems. This a compilation versus interpretation gain. A compilation from full EAGLE to RULER remains to be implemented. 
A prototype Java implementation of RULER, including data (covering integers and object references) and rule-expression parameters, has been developed. The translation algorithm for LTL into RULER has also been implemented, as well as translations from finite automata and regular expressions. The RULER prototype tool has been interfaced as a run-time monitoring system for Java programmes using AspectJ for to instrument the monitored Java code. All examples in the article have been run through the system. Furthermore, the Java integrated RULER tool and tutorial have been used to support a Master's level course on run-time monitoring at Caltech; the students found RULER easy to use and effective. Whilst it is premature to report on case study performance, the prototype

is, so far, supporting our assertions that using RULER rather than EAGLE does lead to more efficient run-time monitoring. Further developments and refinements of the current prototype are in hand and include, for example, hierarchical compositions of RULER monitors, see the tutorial paper [8] for details.

\section{Acknowledgement}

The authors are grateful to Djihed Afifi for producing a parser for the prototype RULER implementation.

\section{References}

[1] R. Alur, K. Etessami and P. Madhusudan. A temporal logic of nested calls and returns. In Proceedings of the 10th TACAS. Vol. 2988 of LNCS, pp. 467-481. Springer, 2004.

[2] J. Baran and H. Barringer. Forays into sequential composition and concatenation in EAGLE. To appear in Proceedings of Run-time Verification Workshop, RV 2008, Budapest, Hungary. Vol. 5289 of LNCS. Springer, 2008.

[3] H. Barringer, M. Fisher, D. Gabbay, R. Owens and M. Reynolds. The Imperative Future: Principles of Executable Temporal Logic. Research Studies Press, Taunton, England, 1996.

[4] H. Barringer, M. Fisher, D. Gabbay, G. Gough and R. Owens. MetateM: an introduction. Formal Aspects of Computing, 7, 533-549, 1995.

[5] H. Barringer, A. Goldberg, K. Havelund and K. Sen. Rule-based runtime verification. In Proceedings of the VMCAI'04, 5th International Conference on Verification, Model Checking and Abstract interpretation, Venice. Vol. 2937 of LNCS, pp. 44-57. Springer, 2004.

[6] H. Barringer, A. Goldberg, K. Havelund and K. Sen. Run-time monitoring in EAGLE. In Proceedings of PADTAD '04, Santa Fe, New Mexico, IEEE Computer Society, IDPDS'04, Vol. 17, No. 17, p. 264b, 2004.

[7] H. Barringer, D. Rydeheard and D. Gabbay. Reactive grammars: an initial exploration. Draft paper, see http://www.cs.man.ac.uk/ david/reactive.html, 2007.

[8] H. Barringer, D. Rydeheard and K. Havelund. RuleR: a tutorial guide. Report available from http://www.cs.man.ac.uk/ howard/LPA.html

[9] G. Brewka. Reasoning about priorities in default logic. In Proceedings of AAAI National Conference on Artificial Intelligence. Vol. 2, pp. 940-945. The AAAI Press/The MIT Press, 1994.

[10] M. D. Fisher. A normal form for temporal logics and its applications in theorem-proving and execution. Journal of Logic and Computation, 7, 429-456, 1997.

[11] B. Finkbeiner and H. Sipma. Checking finite traces using alternating automata. Formal Methods in System Design, 24, 101-127, 2004. 
[12] D. M. Gabbay. Declarative past and imperative future: executable temporal logic for interactive systems. In Proceedings of Coll. on Temporal Logic in Specification, Altrincham. Vol. 398 of LNCS, pp. 67-89. Springer, 1989.

[13] D. M. Gabbay. Introducing reactive kripke semantics and arc accessibility. In Pillars of Computer Science, Vol. 4800 of LNCS, pp. 292-341. Springer, 2008.

[14] S. Greibach. A new normal-form theorem for context-free phrase structure grammars. Journal of the Association of Computing Machinery, 12, 42-52, 1965.

[15] K. Havelund. Runtime verification of C programs. In Proceedings of 20th IFIP TC 6/WG 6.1 International Conference, TestCom 2008 8th International Workshop, FATES 2008, Vol. 5047 of LNCS, pp. 7-22. Springer, 2008.

[16] E. Moriya, D. Hofbauer, M. Huber and F. Otto. On state-alternating context-free grammars. Theoretical Computer Science, 337, 183-216, 2005.

[17] G. Rosu, F. Chen and T. Ball. Synthesising monitors for safety properties — this time with calls and returns. In Proceedings of Run-time Verification Workshop, RV 2008, Budapest, Hungary, Vol. 5289 of LNCS, pp. 51-68. Springer, 2008.

Received 7 April 2008 